\title{
El Dewey Chino: Amigo, demonio y buque insignia
}

\author{
The Chinese Dewey: Friend, fiend, and flagship
}

\section{Le Dewey Chinois : Ami, démon et tête de gamme}

\author{
Barbara Schulte \\ Lunds Universitet, Suecia
}

\section{RESUMEN}

Este artículo analiza cómo se recibió, adaptó y transformó a Dewey en China, en cuatro períodos de tiempo diferentes: durante la era republicana (1912-1949), después de la entrada en el poder del comunismo en 1949, después de que de Deng Xiaoping anunciase las "Cuatro Modernizaciones " (en la década de 1980), y en la China actual. Generalmente, se considera que ha ejercido una tremenda influencia en la educación china. El artículo examina cómo se desarrolló esta influencia tanto a través del propio Dewey como a través de sus mediadores, sus propagadores y sus críticos, entre su visita al país de 1919 y hoy en día. Se presta especial atención a cómo algunas de sus ideas fueron recogidas -y cómo otras fueron ignoradas o tergiversadas- para adaptarse a la agenda de los intelectuales de cada unos de los períodos. Al rastrear los cambios que conceptos centrales como los de "pragmatismo " o " pedagogía centrada en el niño " sufrieron a lo largo de nueve décadas, el artículo revela cómo el filósofo y educador John Dewey fue transformado con éxito en China como 'Duwei' -en amigo del pueblo chino, en demonio de China y el marxismo, y en buque insignia de la modernización.

Descriptores: John Dewey; transferencia educative; procesos de recepción; historia de la educación china.

\section{ABSTRACT}

This article analyzes how Dewey was received, adapted, and transformed in four different time periods in China: during the Republican era (1912-1949), after the Communist take-over in 1949, after Deng Xiaoping's launch of the 'Four Modernizations' (in the 1980s), and in present-day China. Dewey is generally seen to have exerted an immense influence on Chinese education. The article scrutinizes how this influence unfolded both through Dewey himself and through his mediators, propagators, and critics between the time of his visit in 1919 and today. Particular attention is paid to how certain of his ideas were taken up - and others were ignored or twisted - to fit the intellectuals' agenda of each time period. By tracing the changes that central concepts like 'pragmatism' or 'child-centered pedagogy' underwent over the course 
of nine decades, the article reveals how the American philosopher and educator John Dewey was successfully transformed into the Chinese 'Duwei' - into a friend of the Chinese people, a fiend of China and Marxism, and a flagship of modernization.

Key words: John Dewey; educational transfer; reception processes; Chinese educational history.

\section{RÉSUMÉ}

Cet article analyse comment Dewey a été accueilli, adapté et transformé durant quatre différentes périodes de temps en Chine : l'ère républicaine (1919-1949); après la prise de pouvoir communiste en 1949; après le lancement des " quatre modernisations " par Deng Xiaoping (durant les années 1980); et dans la Chine d'aujourd'hui. Dewey est généralement vu comme exerçant une immense influence sur l'éducation chinoise. L'article examine comme cette influence s'est étendue par Dewey lui-même et par ses médiateurs, propagateurs et critiques depuis sa visite en 1919 jusqu'aujourd'hui. Une attention spéciale est portée sur la manière dont ses idées ont été embrassées — et d'autres ignorées ou manipulées — pour correspondre au programme des intellectuels de chaque période. En suivant les changements que des concepts centraux comme " pragmatisme " ou " pédagogie centrée sur l'enfant " ont subis au cours de neuf décennies, l'article révèle comment le philosophe et éducateur John Dewey a été transformé en 'Duwei chinois' - en ami du peuple chinois, en démon de la Chine et du Marxisme, et en tête de gamme de la modernisation.

Mots clés : John Dewey; le transfert éducationnel; les processus d'accueil; I'histoire de l'éducation chinoise.

\section{Introducción: Dewey fluyendo}

$\mathrm{E}$

L TEMA DE “JOHN DEWEY EN CHINA” PARECE SER, con todas las ambivalencias que acompañan a los procesos de transferencia, el primer ejemplo de trasvase oesteeste en educación. La visita de Dewey a China de dos años de duración, entre 1919 y 1921, estuvo precedida por numerosos artículos chinos sobre la educación pragmatista en general y, en particular, sobre sus ideas educativas. Barry Keenan, con su estudio panorámico sobre The Dewey Experiment in China, nos ha proporcionado una amplia información sobre lo que sucedió durante la estancia de Dewey en China, y cómo éste se integró en los debates que por aquel entonces se dieron sobre la reforma educativa, y, de un modo más general, sobre la modernización del país. ${ }^{1}$ En esta contribución, no volveré a contar esta historia. Más bien, voy a ver cómo fue recontextualizado Dewey en China durante un periodo de nueve décadas -desde su visita a China cuando fue recibido como una amigo del pueblo chino, pasando por la era estalinista de los años 1950 donde fue demonizado como el enemigo de China y del marxismo, hasta llegar al aperturismo político chino, que se inició en los años 1970 y a lo largo de los cuales Dewey fue interpretado como el buque insignia de la modernización. En la sección final, presentaré un breve repaso a los debates más recientes sobre Dewey y la educación.

Centrándome en los procesos de recontextualización, voy a mostrar que Dewey no fue simplemente "mal entendido," como un investigador en el pensamiento de Dewey podría percibir de manera intuitiva cuando lee, por ejemplo, los artículos chinos sobre Dewey de los años $1950 .^{2}$ Por el contrario, Dewey -tanto en persona como a través de sus escritos- ofreció un rico repertorio de ideas e ideologías que la intelectualidad china tomó entusiasmada o rechazó con vehemencia para alimentar 
sus argumentos sobre a qué debería parecerse China en el futuro. Recurriendo a las afinidades imaginadas o a las incompatibilidades percibidas, Dewey fue malinterpretado y dado la vuelta para legitimar ciertos objetivos y rechazar otros. Estas afinidades e incompatibilidades hunden sus raíces tanto en los discursos del pasado como en los contemporáneos. Determinadas experiencias colectivas que algunos intelectuales chinos vivieron durante el proceso de modernización del país desde la segunda mitad del siglo XIX en adelante, allanaron el camino para que las "ideas pragmatistas" entrasen en el debate educativo y para que Dewey recibiese una cálida bienvenida a su llegada a China. Sin embargo, también hubo experiencias que despertaron desconfianza o, incluso, hostilidad entre los intelectuales. Estas experiencias contradictorias, que conducían hacia concepciones rivales de la misión educativa que estos intelectuales visionaron para China, se complementaron ampliamente por las experiencias que China tuvo con el marxismo y el maoísmo. Lo que hace que estas experiencias sean tan fascinantes es, una vez más, su ambivalencia: las ideas de Dewey pudieron interpretarse como plenamente coherentes con el marxismo, o como totalmente antitéticas.

Sin embargo, Dewey no solo representó imaginarios conceptuales. A través de su función como profesor y mentor de un gran número de estudiantes chinos, a través de su personal y prolongada visita al país, y, más adelante, a través de su implicación personal en el juicio de Trotsky, no fue sólo la encarnación de unas ideas y teorías más o menos abstractas, sino una personalidad que pudo despertar la admiración, la desconfianza, o, incluso, el odio entre los intelectuales chinos. Ya antes de entrar en China, Dewey no estaba en terreno neutral, sino que formaba parte de redes personales y alianzas ideológicas dentro de los círculos de la élite china. Estaba tan estrechamente unido a sus amigos y mediadores chinos, que a veces es difícil decir quién hablaba a la audiencia china: ¿john Dewey, a través de sus traductores chinos, o sus traductores chinos -todos los cuales eran importantes figuras del ámbito educativo- a través de la prominente personalidad de John Dewey? ${ }^{3}$

Así, las ideas de Dewey se recibieron a través de un prisma de experiencias colectivas, viejas y nuevas, y a través de las alianzas personales de Dewey con determinados agentes del cambio chinos. A este prisma se le añadió una cara más: las ideas de Dewey no sólo fueron "transferidas," ni estuvieron sólo "presentes" durante el proceso de transferencia; Dewey también estaba convencido de que sus ideas eran susceptibles de ser transferidas a otras culturas, ${ }^{4}$ aunque le preocupaba el hecho de los cambios sociales -como el movimiento democrático- que "surgiesen desde dentro." 5 Para él, sus ideas -y su misión- estaban vinculadas a la idea de América en sí misma: “... necesitamos recuperar parte de la militante fe de nuestros padres fundadores, en la cual América es una gran idea, y añadirle una ardiente fe en nuestra capacidad de conducir al mundo hacia la visión de lo que dicha idea significa como modelo para su propio bienestar futuro." ${ }^{6}$ Esta fe de Dewey en el potencial de la tradición (democrática) americana, coincidió con la voluntad de sus seguidores chinos de creer en algo que fuese decididamente diferente a su propia tradición: ante todo, democracia y ciencia. La visita de Dewey en 1919 coincidió con un movimiento que daría voz a estas creencias: el Movimiento del Cuatro de Mayo. ${ }^{7}$

Críticas a Dewey posteriores -principalmente durante la ideologizada era 
estalinista- lo acusaron de hacer de su acercamiento experimentalista una especie de religión ("Religión" era un insulto para los incondicionales del marxismo y el maoísmo). Dada la fe ciega que los antiguos alumnos de Dewey, como Hu Shi (1891-1962) y otros, tenían en las ideas del "pragmatismo," este tipo de críticas se desmontaron fácilmente. El entusiasmo o la crítica hacia John Dewey no sólo tuvieron que ver con las ideas deweyanas. Al menos bajo la superficie, también tuvieron que ver con los conceptos e ideologías chinos que se asociaban con su filosofía y pensamiento educativo. El conflicto que surge entre estas ideologías rivales, tomó en ocasiones la forma de un enfrentamiento entre aquellos que abogaban por la "educación de especialistas" y quienes clamaban por una educación más inclusiva, a menudo insuflada de moral o incluso de inquietudes utópicas. Después de 1949, esto se reformuló como "especialistas" frente a "rojos." La educación china vaciló, yendo y viniendo, entre estos dos extremos, llegando en ocasiones a fases en las cuales los responsables de la política educativa intentaron reconciliar las dos nociones. ${ }^{9}$

Lo que hace que el caso de Dewey en China sea tan interesante, es la constatación de que desde los inicios, el filósofo de la educación americano John Dewey no sólo fue elogiado o detestado, sino que los intelectuales que escribían sobre Dewey siempre tuvieron en mente a "Duwei" - Dewey chino, tal y como fue adaptado en el contexto del país. Por ejemplo, cuando Dewey fue restablecido en China durante los años 1980, no fue sólo por las revisiones de sus escritos, sino también por las ideologías y representantes chinos que había tras ellas. Sin embargo, esto no significa que Dewey representase invariablemente una única ideología o grupo de la intelectualidad china, los conocidos como "rojos" o "especialistas." Al contrario, sus escritos sirvieron a ambas posiciones, e incluso fueron utilizados a veces para reconciliar estas visiones aparentemente contradictorios.

¿Cómo tuvo lugar la adaptación inicial de John Dewey, y cómo se originaron las duras críticas de los años 1950 ?

\section{Dewey y China: el encuentro inicial}

Hoy en día, los investigadores chinos están de acuerdo en que Dewey ejerció una influencia excepcional en el país. No sólo excepcional porque fuese especialmente profunda, sino también porque estos investigadores consideran que fue en China donde Dewey ejerció su mayor impacto - mayor que en cualquier otro lugar, incluyendo a Estados Unidos. ${ }^{10}$ Esta situación de excepcionalidad no puede explicarse solamente a través la personalidad de Dewey. Más bien, tiene que sere relacionada con dos importantes factores: en primer lugar, el "pragmatismo," la palabra clave en el debate chino sobre Dewey en el momento de su visita al país, ha sido un tema candente en la modernización del discurso durante varias décadas. En segundo lugar, John Dewey estuvo relacionado con personas y asociaciones que fueron en sí mismas excepcionales -actores, individuales o colectivos, que, al menos durante cierto periodo de tiempo, parecieron decidir el destino del país. A continuación, desarrollaré estos dos puntos y discutiré después más razones por las cuales Dewey pudo integrarse con tanto éxito en el discurso educativo de la China de aquel tiempo. 
En agosto de 1913, el famoso educador Huang Yanpei (1878-1965), quien por entonces era director de la Oficina de Educación en la provincia de Jiangsu y más tarde fundador de la Asociación China de Formación Profesional, publicó su aclamado ensayo "Debate sobre el uso del pragmatismo en la educación escolar" en un suplemento del Diario Educativo (Jiaoyu Zazhi). ${ }^{11}$ En dicho ensayo, criticaba la tradicional dicotomía entre, por un lado, aprender en la escuela, y, por otro, la realidad y el mundo profesional. Abogaba por una mayor aplicabilidad de lo que se aprendía en la escuela y también por un acercamiento a la enseñanza más centrada en el niño. Todas las escuelas primarias deberían emplear materiales y métodos pragmatistas, y los profesores deberían tomar como punto de partida situaciones de la vida cotidiana. Aunque ésta es una de las primeras veces en que se utiliza el término "pragmatismo," este tema se remonta al siglo XIX, cuando los modernizadores discutían sobre la "utilidad" de la educación. (El término chino para "pragmatismo," shiyongzhuyi, contiene la palabra "útil").

A raíz de la forzada apertura de China tras las Guerras del Opio de mediados del siglo XIX, los modernizadores del país cuestionaron seriamente la "utilidad" de la tradición china. Con "tradición china," lo que tenían básicamente en mente era la tradición de Confucio, esto es, su exagerado énfasis -según estos modernizadores- en los temas morales y estéticos y su negación de las necesidades prácticas de la población. Como consecuencia, estos actores empezaron a buscar un enfoque que acentuase más el "uso práctico" (yong), y que encontraron en una tradición china del siglo XVII, la llamada "estudios concretos" (shixue). Esta perspectiva práctica del mundo consistía en ayudar a los hombres de Estado y a las élites a resolver los asuntos del mundo de manera pragmática (jingshi zhiyong), y se basaba en la asunción de que cualquier tipo de conocimiento debe ser útil para la comunidad o el país.

El argumento del "uso práctico" sirvió de hilo conductor en el discurso de los modernizadores de finales del siglo XIX. El énfasis en el "uso práctico," junto con la creciente conciencia de los modernizadores de las potencias extranjeras, revelaron los problemas inherentes a los sistemas educativos y económicos de China $-\mathrm{y}$ de su íntima relación con los demás. En un comunicado emitido en 1864 por el Ministerio de Asuntos Exteriores, el reformista Li Hongzhang (1823-1901) afirmó que "lo que se necesita no se estudia, y lo que se estudia no se necesita." ${ }^{12}$ Esta frase, que fue repetida en numerosas ocasiones por diferentes reformadores en las décadas siguientes, relacionaba la idea del uso práctico con el problema de los recursos humanos y con la explotación potencial de estos recursos a través de la educación. A través del argumento del "uso práctico," el obsoleto sistema de examen fue críticamente revisado (y abolido en 1905), y se probaron nuevas formas de educación con una fuerte orientación hacia la "aplicación" y el "uso práctico."

Así, el panfleto de Huang sobre la educación pragmatista, de 1913, no fue un fenómeno aislado. De un modo similar, el escritor ya mencionado $\mathrm{Hu}$ Shi, uno de los estudiantes más destacados de Dewey, también recurrió a conceptos tradicionales ya existentes para llegar a su comprensión del "pragmatismo." En sus memorias, recuerda que Dewey lo ayudó a encontrar paralelismos entre la ciencia china y la occidental: 
Él [Dewey; BS] me ayudó a [comprender; BS] los métodos de investigación de la ciencia clásica y de los historiadores desde los últimos mil años -en particular, los últimos trescientos años- como el "kaojuxue," el "kaozhengxue," etc. Traduzco esto al inglés como "investigación evidente," puesto que es una investigación sobre las bases de la evidencia. En su época sólo había unas pocas personas (prácticamente ninguna) que hiciese notar las similitudes que existían entre los principios de la ciencia moderna y nuestro clásico método kaoju, kaozheng. Yo fui el primero en haber realizado estas declaraciones. Y la razón por la cual pude hacerlo fue, en realidad, gracias a las teorías de Dewey. ${ }^{13}$

En consecuencia, Hu Shi usó el término shiyanzhuyi (literalmente: "experimentalismo"), ${ }^{14}$ en lugar del término más común "pragmatismo." Sin embargo, al igual que Huang Yanpei, favoreció el "pragmatismo" por encima del aprendizaje clásico, que veía como "mucho ruido y pocas nueces:" se discutían cosas abstractas que no tenían ninguna relevancia, de lo cual uno sólo podía salvarse si empezaba a mirar cosas concretas. ${ }^{15}$ Pero, a diferencia de Dewey, Hu Shi no tenía motivos por los que le preocupase una visión excesivamente simplificada de la ciencia. En el contexto chino, vio que la superstición, y no el cientificismo, era el verdadero enemigo de la ciencia moderna. El "pragmatismo" era un arma para luchar contra la superstición - $e l$ arma. ${ }^{16}$ Tal y como señaló Sor-Hoon, Hu Shi cambió la frase de Dewey de "el pragmatismo debe tratarse principalmente como un método," en la afirmación "Dewey, de principio a fin, sólo reconocía al pragmatismo como un método." ${ }^{17}$ Así, Hu convirtió el "pragmatismo" en un concepto extremista que hizo imposible cualquier tipo de compromiso por parte de los reformistas más moderados. Para él, el pragmatismo estaba intrínsecamente unido con la ciencia occidental, y, a través de ella, a la superioridad de Occidente:

Condeno sin reservas nuestra civilización oriental, y acojo con gran admiración la moderna civilización occidental. En ocasiones se ha dicho que la civilización oriental es idealista mientras que la de Occidente es materialista. Esto es una falsedad fabricada por personas que sufren egoístas ilusiones... Hay cretinos... que desean que creas que la antigua cultura china y los valores morales son superiores a todos los demás. También hay idiotas que, no habiendo estado nunca en el extranjero, gritaban: "iAl Este! ¡Al Este! La trampa de Occidente ya no funciona." Lo que quiero deciros es que no os dejéis engañar. Debemos admitir... que somos inferiores a los otros no sólo en tecnología e instituciones políticas, sino también en cuanto a valores morales, conocimiento, literatura, música, bellas artes y aspecto físico. ${ }^{18}$

A través de esta interpretación extrema del "pragmatismo," Hu Shi quedó instalado en una precaria situación. No sólo los tradicionalistas -o, más tarde, los neo-tradicionalistas como el filósofo Liang Shuming (1893-1988)- se oponían con fuerza a lo que ellos entendían como una negación de la propia cultura; sino que también otros intelectuales, independientemente de su orientación política, fueron críticos con una 
disposición tal a adorar los valores occidentales. Hubo además numerosas ocasiones en las que Dewey advirtió contra la erradicación de las estructuras tradicionales, como las de clan familiar o gremio, sin equivalentes capaces de sustituirlas. ${ }^{19} \mathrm{La}(\mathrm{re})$ presentación extrema que de Dewey hizo Hu Shi, podría haber hecho especialmente fácil para los críticos atacar a Dewey, ya fuese desde una perspectiva tradicionalista o marxista. Algunos incluso acusaron a Hu de "'secuestrar' a Dewey, de tratarlo como un 'títere', y de utilizarlo para 'aumentar su propia fama' y 'destruir por completo la cultura tradicional china.'”20

No obstante -y ahora con respecto al segundo punto de la conexión de Dewey en China- la personalidad de Hu Shi fue una de las razones por las cuales Dewey pudo llegar una audiencia tan vasta. Hu Shi y otros mediadores de Dewey en China fueron, todos ellos, bien conocidos por su agudeza intelectual y su talento retórico. A la población culta le gustaba mucho escucharlos y acudía a sus conferencias como si fuesen a ver a una estrella del pop.

El carisma de hombres como Hu Shih, produjo un efecto en las conferencias de Dewey que, de otro modo, el casi sexagenario no habría conseguido sobre los estudiantes. [...] Las conferencias de Dewey, así como sus libros en inglés, no estaban claras para la audiencia china-pero Hu Shih era un excelente orador y su interpretación era comprensible para todo el mundo. ${ }^{21}$

Al mismo tiempo, el tour de Dewey por China estuvo meticulosamente orquestado. Una vez más, la comparación con el tour de una estrella del pop no parece exagerada. Los mediadores de Dewey se aseguraron de que sus conferencias recibiesen una extensa cobertura de los medios de comunicación. Dos periódicos, Nueva Juventud y El Diario de la Mañana, informaron de cada uno de los eventos que tenían que ver con Dewey; no sólo publicaron sus conferencias, sino que también reprodujeron pequeños coloquios, fotografías y similares. ${ }^{22}$ La revista educativa Nueva Educación, fundada por otro famoso alumno de Dewey, Tao Xingzhi (1891-1946; por aquel entonces Decano de la Facultad de Educación del Centro del Profesorado en Nanking) admiraba tanto las teorías educativas de Dewey, que Hu Shi lo llamó la "revista deweyana." 23 Puesto que la publicación era ampliamente leída en el ámbito académico chino, la audiencia de Dewey debía saber lo que tenía que decir (o lo que sus mediadores tradujeron). Junto con la cobertura que los medios hicieron de sus actividades y conferencias -Dewey ofreció alrededor de setenta y ocho conferencias en diferentes foros alrededor de todo el país, constando algunas de ellas de quince o veinte lecciones cada una- sus conferencias traducidas se publicaron como libros. Cuando Dewey dejó China, una editorial de Beijing ya había vendido una docena de ediciones de sus conferencias más importantes, siendo cada edición de alrededor 10000 copias (iy cada copia contenía unas 500 páginas!). ${ }^{24}$ Muchas de sus obras que ya habían sido traducidas - por encima de todas, Democracy and Education- fueron empleadas como material de lectura en las facultades y universidades.

La expectación que él y sus conferencias despertaban no dejó de impresionar a Dewey, quien consideraba estar "en manos de la joven China ... Debemos pasar un 
rato muy bueno. Muy diferente a Japón," que parecía "la tierra de los miramientos y las reticencias." ${ }^{25}$ A diferencia de lo que pasó en Japón, Dewey se consideró firmemente integrado en la intelectualidad china. Además, tuvo acceso a algunas de las asociaciones más relevantes del país dado que sus antiguos alumnos habían ocupado puestos importantes no sólo en instituciones de la importancia de la Universidad de Beijing y el Centro de Formación de Profesorado de Nanjing, sino también en las organizaciones que determinaban en buena medida la política educativa de la época, y a menudo la política misma. Lo que el alemán Carl Heinrich Becker, quien en 1931 lideraba la Liga de la Comisión de la Educación Nacional de China, llamó una "devastadora" influencia sobre el Centro de Formación del Profesorado de China, ${ }^{26}$ puede reformularse en un sentido positivo como el amplio potencial para determinar la política que estaba en manos de los alumnos chinos del Centro de Formación del Profesorado. A través de sus contactos, Dewey fue capaz de participar directamente en los debates y las propuestas de reforma. Habló en las asambleas nacionales de numerosas organizaciones educativas que entre 1915 y 1922 trabajaron en el diseño de un nuevo sistema educativo, que finalmente sustituyó al antiguo en 1922. (Este sistema fue más adelante tildado de "americanizado"). Mientras que Dewey seguramente daba la impresión de poder acentuar el proceso de reforma, sus apariciones en dichas asambleas -así como en otras instituciones- también pueden interpretarse como intentos, por parte de sus mediadores chinos, de utilizar su preeminencia y popularidad para la persecución de sus propios intereses. Dewey servía como un efectivo sello de calidad si decía estar de acuerdo con ciertas propuestas de reforma o si visitaba determinada escuela. Y su aprobación -o simple presencia- era especialmente necesaria si se trataba de una propuesta o institución controvertida. Por ejemplo, se informó de su visita a una escuela primaria donde se había introducido la artesanía como asignatura, ${ }^{27}$ así como de su reconocimiento a los logros de la Escuela de Formación Profesional China de Shangai. ${ }^{28}$

Así, los antiguos alumnos de Dewey y por ello mediadores, tenían un interés vital por asegurar que Dewey mantuviese su elevada reputación y amplia popularidad. Tal y como apunta Keenan, debieron influir también en la opinión americana sobre los acontecimientos chinos, puesto que los análisis de China hechos por Dewey que se publicaron en los medios estadounidenses, se basaban en gran medida en las opiniones que sus antiguos alumnos tenían, por ejemplo, del movimiento democrático chino. En este sentido, Dewey subrayó en repetidas ocasiones que estaba en deuda con un "buen amigo chino" en su análisis de la situación china -es muy probable que este amigo fuese $\mathrm{Hu} \mathrm{Shi}^{29}$

Una coincidencia más hizo que la estrella de John Dewey se elevase aún más en China: su sesenta cumpleaños el 20 de octubre de 1919, coincidió, según el calendario lunar, con el $2470^{\circ}$ cumpleaños de Confucio. El famoso Cai Yuanpei (1868-1940), un antiguo alumno de la Universidad de Leipzig y Ministro de Educación chino desde la fundación de la República en 1912, no se equivocó al señalar las similitudes entre las dos figuras, refiriéndose a Confucio y acentuando la importancia de la experiencia: "Aprender sin pensar es un desastre; pero pensar sin aprender es peligroso." Sin embargo, también señaló las diferencias, elevando así a Dewey sobre Confucio: 
Confucio dijo respetad al emperador (wang), el sabio doctor (poshih) defiende la democracia; Confucio dijo que las mujeres son un problema en aumento, el sabio doctor abogó por iguales derechos para hombres y mujeres; Confucio dijo transmitid y no creéis, el sabio doctor abogó por la creatividad. ${ }^{30}$

A través de tan halagadora introducción venida de una persona tan famosa y respetada, John Dewey apareció no sólo como una alternativa a Confucio, sino como una especie de Confucio alternativo en sí mismo: un sabio y maestro de la mentalidad moderna que sería ampliamente escuchado en China.

Pero, ¿cuáles fueron los temas - tras el concepto general de "pragmatismo"- que de hecho se escuchaban? y ¿cómo se relacionaron con las agudas problemáticas de las élites chinas? Tres grupos temáticos destacan por haber recibido mayor atención en el debate republicano chino de inspiración deweyana: el debate sobre la ciencia y la salvación de la nación, el argumento contra el "viejo estilo de educación" y el descubrimiento del niño y la discusión sobre la formación profesional y de masas.

Para muchos chinos, Dewey era sinónimo de modernidad, "Occidente," ciencia. Una vez más la ciencia occidental -o un sistema educativo que estuviese modelado según los estándares de la ciencia occidental- se consideró necesario para la supervivencia del país y de su pueblo - "la educación para salvar el país" (jiaoyu jiuguo). Al igual que el discurso sobre el "pragmatismo," el discurso sobre la salvación-de-la-nación volvió al siglo XIX y desveló su inspiración en el darwinismo social. Hizo que la convicción de Dewey acerca de que la educación -y no la revolución- es lo que haría mejorar al país, fuese mucho más convincente. Desde este antiguo hilo discursivo sobre la ciencia, la educación y la supervivencia de la nación, llegó la cientificación de la educación -o, en palabras de Carl Heinrich Becker, "la disolución de todo conocimiento real en sandeces sobre metodología y psicología de la educación." ${ }^{11}$ Con su punto de vista sobre la pedagogía centrada en el niño, sus teorías sobre la inteligencia humana, y sus experiencias prácticas a través de los laboratorios escolares, Dewey era el ideal representativo de esta nueva tendencia en educación; los altos puestos que sus antiguos alumnos alcanzaron tras regresar a China probablemente reforzasen este enfoque. El rol de la educación como un cúralo-todo de las dolencias nacionales parecía ahora mucho más refinado: mientras que en el sentido tradicional, principalmente destinado a la formación de talentos y la educación moral, los métodos recientemente introducidos desde la psicología prometían proporcionar herramientas mucho más eficientes y científicas para comprender y manipular al ser humano, y en particular, la mente del niño. ${ }^{32}$

El viejo estilo de educación, tal y como ha sido señalado arriba con respecto al debate sobre el "pragmatismo," ya fue criticado en el siglo XIX. El aprendizaje memorístico y el aprendizaje exclusivamente orientado hacia los exámenes (para convertirse en funcionario) se encontraron con críticas especialmente duras. Naturalmente, el rechazo de Dewey a una educación totalmente desconectada de la realidad del niño, fue recibido con entusiasmo por los reformistas intelectuales chinos. Sin embargo, lo que más adelante avivó el debate fue el descubrimiento del niño por parte de los chinos, lo que coincidió con la recepción en el país de la pedagogía centrada en el 
niño del movimiento de la educación progresiva, de la que Dewey era considerado el máximo exponente. Durante el Movimiento del Cuatro de Mayo -que pasó a conocerse entonces como la Revolución Cultural China- el niño se convirtió de pronto en el centro de atención: no sólo como la personificación del futuro de la nación, sino como un ser humano con derecho propio. ${ }^{33}$ Cierto número de escritores atacaron la visión tradicional inhumana de las personas en general y del niño en particular, donde era visto como una especie de forma adulta aún sin desarrollar. Uno de los escritores chinos modernos más famosos, Lu Xun (1881-1936), atacó este tradicional error cometido contra la infancia en su artículo "Cómo seremos padres ahora." 34

Educadores inspirados en la concepción del niño de Dewey, empezaron a hablar del "innato" deseo infantil por construir, y sobre cómo podría convertirse en algo útil para la educación escolar. Ahora se esperaba que los profesores fomentasen la "imaginación creativa" de los estudiantes, y fortaleciesen su "espíritu constructivo," mientras que antes se les encomendaba el seguimiento de los avances de los estudiantes en el aprendizaje memorístico. Tomando las ideas de Dewey, el concepto de ciencia no sólo se fusionó con su aplicabilidad, sino también con la psicología del niño, la singularidad de cada resultado (producido durante el proceso de aprendizaje), y la libertad de expresión cuando se crea algo nuevo. ${ }^{35}$

El último grupo temático -la formación profesional y la educación de masas- representa también, al igual que los temas ya discutidos, la coyuntura de antiguos y nuevos hilos discursivos. El movimiento de la formación profesional tuvo sus precedentes en el siglo XIX, cuando en las fábricas de nueva creación surgió la necesidad de personal capacitado. La formación profesional fue principalmente concebida como la “educación de especialistas." La recepción del enfoque de Dewey sobre la formación profesional coincidió con un cambio de enfoque dentro del movimiento chino de la formación profesional. La formación profesional no era ya sólo una solución a los problemas del país para encontrar mano de obra adecuadamente capacitada. Más bien, era la solución al problema más urgente del país: el problema de cómo ganarse la vida (shengji wenti). La formación profesional se convirtió entonces en un fenómeno de masas y se incorporó a los movimientos para extender la educación alrededor de todo el país (pingmin jiaoyu). Cuando advertía por medio de sus conferencias de que no se olvidasen las zonas rurales, tocó la fibra sensible de los intelectuales chinos, que se habían dado cuenta de cómo los programas de reforma educativa se habían centrado hasta el momento en las zonas urbanas. Hoy, los investigadores chinos sostienen en general la opinión de que la visita de Dewey y sus conferencias inspiraron a la intelectualidad china para que extendiese los esfuerzos de la reforma a las áreas rurales. ${ }^{36}$ Sin embargo, muchos escritores y educadores reformistas ya habían prestado atención a la situación del campo en China en el momento de la visita de Dewey -motivados por la búsqueda de malos hábitos y supersticiones nocivas o alarmados por los resultados de la evaluaciones educativas, las cuales, en el transcurso de la cientificación de la educación, fueron siendo cada vez más populares. La visita de Dewey puede verse como un refuerzo y una mayor legitimación de los esfuerzos de estos intelectuales para educar las zonas rurales.

Con la aparición de la formación profesional y la educación de masas, la raison 
d'être de la educación general empezó a ser severamente cuestionada. Con su oposición a la separación de la educación general y la formación profesional, Dewey sirvió como punto de referencia. Por ejemplo, el más arriba mencionado fundador de la Asociación China de Formación Profesional, Huang Yanpei, vio toda la educación permeada de formación profesional. Dewey, argumentaba Huang, lo vio como el principio fundamental de la educación que el individuo podía recibir para poder ganarse la vida a la vez que sirve a la sociedad; éstos eran, según afirmó Huang, exactamente los objetivos de la formación profesional. En este sentido, no había una educación general: toda forma de educación se relacionaba con algún tipo de profesión, y cada profesión con algún tipo de educación. ${ }^{37}$ Asimismo, Wang Maozu (1891-1949), propagador de la educación de masas dentro de la Asociación, señaló que lo que se entendía por "formación profesional" debía ser, en realidad, una parte integral de cualquier tipo de educación. Si todas esas cosas que se consideraban útiles, prácticas y relevantes para la vida profesional se incluyesen en la "formación profesional," “¿en qué consistirá entonces la educación general? ¿Significa que la educación general se limita a una educación donde uno maneja unas pocas características oscuras e incomprensibles?" 38 Si la misión de la educación era preparar a los estudiantes para la vida, entonces, al final, cualquier clase de educación era algún tipo de formación profesional. La afirmación de Dewey (también pronunciada durante una conferencia en Shangai) de que "las educación era la vida misma," fue muy bien acogida. El trabajo, la vida y la educación parecían estar ahora confusamente unidos. ${ }^{39}$

Tal y como hemos mostrado, la República China fue muy receptiva a las ideas de Dewey -de nuevo, muy diferente al caso de Japón, donde el reinado hegemónico del modelo alemán/tradicional fue un obstáculo para cualquier toma en consideración de las ideas de Dewey. ${ }^{40}$ Es interesante que Dewey fuese capaz de satisfacer las necesidades de las fuerzas progresistas y de las conservadoras moderadas. Sus convicciones democráticas le hicieron ser atractivo para los seguidores del Movimiento del Cuatro de Mayo, pero al mismo tiempo sus objeciones acerca de la revolución y el uso de la violencia lo hicieron digerible para quienes querían reformar y transformar el país a través de la educación. Muchos, interpretaron incluso el punto de vista de Dewey sobre los estudiantes y (futuros) trabajadores como una garantía de estabilidad social: a los estudiantes se les enseñaba a contentarse con su situación y sacar lo mejor de ella, en lugar de darle la vuelta, tal y como reclamaban las fuerzas más radicales. El mensaje básico de la obra de Dewey Democracia y Educación, siguiendo estas razones, se transformó en el principio de que "cada uno debe estar en su lugar oportuno" y "cada uno debe estar oportunamente preparado para ello." ${ }^{41}$ Los propagandistas de la formación profesional fueron especialmente propensos a seguir esta línea argumental, que fue criticada por contemporáneos como el anteriormente mencionado discípulo de Dewey Tao Xingzhi, por ser el "principio de ropa y alimentos" (yishizhuyi). Como veremos, este "principio" fue ferozmente atacado en la década de 1950.

Sin embargo, la amistad de Dewey con China no careció de fallas, como el hecho de que reciviese algunas críticas antes de 1949. John Dewey encontró resistencia entre los marxistas y entre quienes estaba preocupados por una extremada occidentalización o "copia." Este último grupo no estaba ideológicamente definido y estaba 
compuesto por neo-tradicionalistas así como por reformistas y marxistas moderados. $\mathrm{Su}$ argumento básico era que China no debía cometer el error de copiar ciegamente de otros países - un argumento que ya había surgido en los inicios de la República ${ }^{42}$ y que se recordó repetidamente en la época comunista (incluidos los debates reformistas más recientes). Sólo deberían tomarse prestadas cosas de otros países bajo criterios sensatos que eviten el error anterior de "absorber la civilización de otros países para satisfacer las propias necesidades;" "inflar los resultados eficientes del extranjero" sólo llevaría a mayores "errores y desventajas que caerían a cántaros" sobre China. ${ }^{43}$ Los educadores advirtieron de que China no debía negar su propia historia y sociedad:

Dado que durante las reformas quienes eran políticamente responsables sólo buscaban fortalecer [el país; BS], nadie preguntó por qué Japón podía copiar a Alemania; la gente sólo quería destruir, en el menor tiempo posible, la historia china, destruir el entorno social y convertir una sociedad agraria micro-estructurada en una sociedad definida por la industria y el comercio. ${ }^{44}$

A pesar de que, como hemos señalado, no era en absoluto la intención de Dewey "des-culturizar" a China, las experiencias previas junto con la copia a ciegas hicieron que algunos reformistas fuesen cautos a la hora de abrazar su teoría. Otra razón podría haber sido la proliferación de "laboratorios escolares" (shiyan xuexiao) de inspiración deweyana, de entre los cuales no todos tenían la calidad que los educadores deseaban que tuviesen. En un crítico artículo sobre estas escuelas, Zhao Yichen preguntaba sarcásticamente: "Por lo que respecta al carácter del laboratorio ¿podría ser el laboratorio fuese el método de copiar a América?" 45

Una de las primeras críticas desde el punto de vista marxista, de acuerdo con Jessica Wang, la articuló en 1921 el comunista Fei Juetian, quien sostuvo que Dewey se preocupaba demasiado de las especulaciones abstractas y muy poco de los hechos concretos. ${ }^{46}$ Otros marxistas sostuvieron que el pragmatismo de Dewey sólo beneficiaba a la burguesía. En educación, el pedagogo Jiang Qi (1886-1951) atacó el intentó de Dewey de eliminar la diferencia entre la "formación profesional" y la "educación liberal," o la "educación profesional" y la "educación cultural." Citando extensamente a Marx y a Sun Yatsen, Jiang argumentaba que la formación profesional, desde una perspectiva histórica, existió mucho antes que la "educación liberal;" cumplía con la tarea original de desarrollar las fuerzas de producción social (gesellschaftliche Produktivkräfte), con las que la "educación liberal” no tenía nada que ver. Negar la diferencia entre estos dos tipos de educación no haría justicia a la importancia fundamental de la formación profesional. Sin embargo, Jiang estaba de acuerdo con Dewey a la hora de aceptar el término "formación profesional" (y sustituir el de "educación para la producción" (shengchan jiaoyu)), dado que la "formación profesional" podía entenderse fácilmente como una "educación con fines de lucro" (yingli jiaoyu). En un movimiento final, Jiang aceptó que las ideas de Dewey debían usarse en China mientras ésta estuviese en su etapa capitalista, puesto que Dewey y China seguían siendo "extremadamente conservadores." ${ }^{47}$ Esta crítica puede tildarse casi de suave, comparada con la que llegaría en la década de 1950. 


\section{Un estafador y un charlatán: Dewey en la era marxista}

Los primeros comunistas no temían ponerse en contacto con Dewey. Al contrario, Dewey se llevaba bien con dos de los padres fundadores del comunismo chino, Chen Duxiu (1879-1942) y Zhou Enlai (1898-1976). Incluso Mao Zedong (1893-1976) asistió a dos conferencias de Dewey en Shangai y Changsha. En Changsha, en octubre de 1920, el diario de Hunan Da Gongbao encargó a Mao que tomase notas de la conferencia de Dewey. Según Lu Guoqi, Mao se refirió explícitamente a Dewey en sus primeros pensamientos educativos y estuvo claramente influenciada por las ideas de Dewey, a pesar de que al mismo tiempo reconocía su ignorancia a la hora de reconocer las divisiones ideológicas: "Para ser honesto, sigo sin tener claros todos estos -ismos y teorías diferentes." 48

Esta pacífica coexistencia de ideologías finalizó abruptamente con la caída del estalinismo. Dewey era ahora claramente identificado como persona non grata y un enemigo de la clase. Su antiguo alumno Chen Heqin (1895-1982) fue forzado a participar públicamente de la auto-crítica (renzuishu) y a denunciar a Dewey como "reaccionario," como un representante del "idealismo subjetivo" y como el "mayor hipócrita y estafador en la historia de la educación.” ${ }^{49}$ A través de su hipocresía, tal y como argumentaban sus crítico, Dewey había sido capaz de engañar a un gran número de honrados marxistas, al haberse disfrazado de "progresista" y "de izquierdas." Esto hizo que su crimen -ser un contra-revolucionario y capitalista reaccionariofuese el más grave. Como la vanguardia del capitalismo, Dewey se convirtió en el blanco de toda una serie de ataques feroces, o como uno de sus principales críticos, Cau Fu, ${ }^{50}$ señaló: "Si se quiere disparar a un hombre, hay que disparar primero al caballo.” ¿Qué es lo que este caballo llevaba sobre sus espaldas que molestaba tanto a sus críticos?

Como hemos indicado más arriba. Dewey se opuso abiertamente a la revolución como medio de transformar China; manteniéndose estrictamente contrario al uso de la violencia. Desde un punto de vista marxista, sustituir la revolución por la educación era, en el mejor de los casos, una ingenuidad. También se consideraba injusto en dos aspectos: en primer lugar, el propio Dewey se beneficiaba de antiguas revoluciones violentas que dieron lugar al liberalismo - ¿por qué no aceptaba los mismos medios para alcanzar el socialismo? En segundo lugar, la clase dirigente capitalista que era, irónicamente, experta en el uso de la violencia y la represión sólo sería expulsada en el campo de batalla, y no en el parlamento: "No es que el proletariado no quiera usar el 'método de la inteligencia', sino que la clase dirigente no les permite usar el 'método de la inteligencia."'51 Pero, peor aún, muchos críticos simplemente no creían en la ingenuidad de Dewey cuando hablaba en contra de la revolución. Dewey era presentado tan astuto como para disfrazar sus objetivos reales pretendiendo ser un educador ingenuo; en realidad, él formaba parte de una conspiración que pretendía mantener a los trabajadores ignorantes y avergonzados. ¿Cómo pudieron sus críticos llegar a tal conclusión, y qué bagaje ideológico se consideraba especialmente incompatible con la manera marxista de ver el mundo?

Claramente, la objeción de Dewey a la revolución era sólo una razón -pero no 
la razón principal- por la que los marxistas no podían aceptar su pensamiento. En el fondo de la obra de Dewey los marxistas chinos veían propuestas que, tomadas seriamente, hubiesen destruido la cosmovisión chino-marxista. En la discusión sobre Dewey, tres grandes temas aparecieron una y otra vez: el "naturalismo," la supuesta posición "anti-científica" de Dewey y su proposición acerca de la "incertidumbre."

Ya en 1950, Dewey fue acusado de "naturalismo" o "biologicismo" -por la aplicación demasiado simplista del conocimiento biológico al mundo social. Sus críticos lo atribuyeron a su orientación darwinista: el progreso y el desarrollo eran presentados como un fenómeno innato y biológico que podía y debía no ser manipulado o acelerado a través de las fuerzas sociales. A principio de los años 1950 se consideró que esta posición era insostenible, dado que Marx demandaba claramente que la educación promoviese el desarrollo humano en una determinada dirección: “ ¡Evidentemente, el cambio social sin una dirección u objetivo significa que la sociedad no cambia!" 52 A mediados de los años 1950, esto fue reformulado de una manera más polémica: cualquier asunción basada en un "talento innato" era, en primer lugar, equivocada porque el individuo era un producto de la sociedad. En segundo lugar, términos como "talento innato" eran sospechosos de ser usados para justificar por qué los trabajadores estaban subordinados a la clase capitalista al ser considerados como carentes de "talento innato." ${ }^{53} \mathrm{Al}$ igual que con su postura en contra de la revolución, Dewey fue presentado como parte de una gran conspiración para mantener el sistema capitalista. Asimismo, el tono de la argumentación se hizo más ofensivo: las obras de Dewey eran descritas como un "reflejo del monopolio, podrido, la etapa moribunda en la que el capitalismo ha entrado;" sus seguidores chinos como Hu Shi o Chen Heqin fueron llamados "perros ejecutores" (zougou); Dewey debía ser "purgado" de China. ${ }^{54}$

La postura aparentemente "anti-científica" de Dewey era otro aspecto de la crítica. En la superficie, ésta no parecía amenazar los fundamentos de las creencias marxistas. Sin embargo, depende de cómo se defina "ciencia" -o "anti-ciencia." En un primer paso, la teoría de Dewey fue reconstruida; fue descrita como si estuviese solamente basada en la psicología, negando inexcusablemente el contenido científico y lógico. Irónicamente, Cao Fu usó la misma cita de Confucio que Cay Yuanpei empleó cuando dio la bienvenida a Dewey en China: "Aprender sin pensar es un desastre; pero pensar sin aprender es peligroso." 55 Dewey fue acusado de deshacerse de todo el conocimiento científico que se había ido acumulado a lo largo de los siglos, sólo para dejar ser a los niños pequeños "descubridores" e "inventores;" sustituyendo el currículo orientado a la disciplina por el trabajo por proyectos, los estudiantes nunca adquirirían un conocimiento sistemático de las diferentes ciencias. Aún más chocante para sus críticos era el hecho de que los profesores fuesen despojados de toda autoridad: "se niega la función principal del maestro." ${ }^{56}$ Chen Wenbo apeló dramáticamente al lector para que no olvidase al profesor como el "guerrero positivo de la humanidad en la batalla contra la ignorancia." ${ }^{57} \mathrm{Cao} \mathrm{Fu}$, citando a Pedro T. Orata, expresó su temor de que este tipo de método de enseñanza sólo conduciría al caos, o incluso al anarquismo. ${ }^{58}$ En cuanto a la pedagogía centrada en el niño de Dewey, resumió el punto de vista chino-marxista de un modo conciso: 
No necesitamos usar el deweyanismo como un arma, puesto que parte del antiformalismo de la metodología de Dewey se construye sobre la base equivocada del principio centrado en el niño. El principio centrado en el niño es individualista, y nos oponemos al individualismo. [...] ¡ Debemos adoptar la avanzada experiencia soviética para construir un país y de ahora en adelante debemos fortalecer la ardua formación de los conocimientos científicos sistemáticos en las escuelas! $!^{59}$

El segundo paso en la acusación de anticientificismo contra Dewey, fue mucho más lejos, situando al autor como una seria amenaza al principio esencial del marxismo: el principio de que había una verdad objetiva que podía alcanzarse mediante métodos científicos. Los marxistas chinos fueron muy críticos con la relativización de la verdad que hacía Dewey. Zhong Chengzhang, en un artículo de 1963, lanzó un ataque contra el "pragmatismo," que sostenía que "lo que es útil es verdad." Expresó su indignación por la declaración de Hu Shi acerca de que la verdad sólo era un producto de los seres humanos. Esta idea fue considerada sumamente egoísta y carente de toda moral: la relatividad de la verdad se conectó entonces con el "individualismo" y con un capitalismo "egoísta" y "de fines lucrativos." ${ }^{60}$ Éste fue un paso más hacia la pérdida de valor y la progresiva desespecialización del uso del término pragmatismo: el "pragmatismo" fue convirtiéndose cada vez más y más en un sinónimo de "egoísmo."

Esta acusación de negar una verdad objetiva lleva directamente al tercer y último gran tema en el debate sobre Dewey de los años 1950 y 1960: la propuesta deweyana de la "incertidumbre." Al igual que con el tema anterior, la teoría de la "incertidumbre" fue atacada desde dos ángulos: por un lado, la incompetencia de Dewey debía demostrarse desde un punto de vista científico; por el otro, la "incertidumbre" era vista como una amenaza existencial para el marxismo. Desde un punto de vista científico, la afirmación de Dewey de que la educación "carecía de fines definidos," fue ridiculizada como un fracaso en el reconocimiento del objetivo de la educación, que era, por supuesto, la integración del individuo en la sociedad (socialista). Un reproche estrechamente relacionado con éste, era que este tipo de "educación sin fines definidos" no tenía ninguna base ideológica y, por lo tanto, no establecía normas acerca de lo "bueno" y lo "malo." Como Cao Fu señaló, una teoría de la educación como ésta podía servir tanto a una sociedad socialista como a una fascista; su vaguedad en términos ideológicos hubiese conducido a la despolitización de la escuela y, por consiguiente, a la ignorancia política de los estudiantes. ${ }^{61}$

Una vez más, los críticos de mediados y finales de los años 1950 fueron más severos en sus opiniones: la educación "sin fines definidos" de Dewey no era sólo una teoría equivocada, sino que de hecho estaba deliberadamente dirigida a perpetuar la ignorancia de los trabajadores. Redirigiendo la atención de los niños solamente hacia las cosas más básicas (la llamada "pedagogía centrada en el niño"), estarían preparados para convertirse en trabajadores capaces de "proporcionarse ropa y alimentos." A través de esa educación tan estrictamente restringida, los estudiantes no tendrían acceso a las teorías y conocimientos sistemáticos que les permitirían emanciparse. Los estudiantes habían sido, por lo tanto, literalmente privados de los instrumentos que 
hubiesen podido iluminarlos, como los maestros y los libros: "Si esto no es engañar a la gente, entonces, ¿qué es?" ${ }_{2}$ La teoría de Dewey "de la educación sin fines definidos" fue considerada como "un cuento de hadas propagado por la clase capitalista según el cual la educación está aparte de la política, es una especie de intriga vil en el que ellos [los capitalistas; BS] planean encubrir el objetivo reaccionario de la educación imperialista." ${ }^{63}$ Atacar a Dewey en estos aspectos significaba también atacar a los educadores, tanto del pasado como los contemporáneos, que considerasen que lo más importantes para los trabajadores era satisfacer sus necesidades fundamentales: protegerse del hambre y del frío. Esta idea más bien tecnocrática de la misión educativa no tenía cabida en el cada vez más utópico mundo de la década de 1960.

Sin embargo, la denuncia de la "incertidumbre," no sólo afectaba a la falta de fines definidos de la educación. Para la cosmovisión chino-marxista, significaba mucho más. Paralelamente a la enfurecida refutación de que la verdad fuese subjetiva y relativa, la teoría de la "incertidumbre" de Dewey se encontró con una resistencia similar, ya que implicaba que el curso de la historia como tal -y por lo tanto el futuro- eran inciertos. Esto contradecía directamente la idea marxista de las inevitables etapas históricas, y también amenazaba peligrosamente la capacidad de construir el mundo. Tal y como Jin Yuelin razonó, "somos capaces de reconocer las leyes objetivas e inevitables, pero por otra parte, a través de nuestro correcto reconocimiento, somos capaces de reconstruir el mundo." ${ }^{64}$ Como si se estuviese consolando a sí mismo y a sus lectores, explicaba entonces que la razón por la cual Dewey transmitía la imagen de un mundo inestable e inseguro, era que el propio sistema capitalista estaba al borde del colapso; para los capitalistas, el mundo era, en efecto, inestable y peligroso. Cao Fu convirtió su discurso contra la incertidumbre deweyana, prácticamente en una súplica: "la filosofía marxista y leninista nos ha dado un objetivo 'cierto', una dirección correcta. El pueblo chino cree firmemente en este objetivo y se mantiene en esta dirección, en consecuencia, ha encontrado finalmente la salvación." ${ }^{65}$ De pronto parecía que había mucho que perder si se seguía a Dewey.

Durante la Revolución Cultural de 1966 a 1976, Dewey fue silenciado casi por completo. A primera vista, esto no es sorprendente: como un pensador occidental de la ideología equivocada, no tenía lugar en el discurso de la Revolución Cultural. Sin embargo, si miramos más detenidamente, es extraño que Dewey estuviese casi totalmente ausente del discurso, incluso como enemigo de clase. Hay un artículo de 1974 - ¿Quién dice que una educación abierta es 'Pragmatismo?" "66- que puede darnos una pista muy clara acerca de por qué esto es así: había demasiadas -aunque superficialessimilitudes entre las prácticas educativas de Dewey y las de la Banda de los Cuatro. En su artículo, Juan hizo alusión al hecho de que "algunas personas," habían defendido que la idea de la "educación abierta” tenía algunos puntos en común con la "educación pragmatista" de Dewey. Pero luego se esforzó por demostrar que una no tenía nada que ver con la otra: los profesores de la educación abierta eran campesinos, obreros y soldados; y, además, el "trabajo por proyectos" de Dewey estaba muy lejos del mundo real, mientras que los revolucionarios culturales salían de verdad al mundo real. Estas fueron las dos razones más concretas por las cuales Juan pudo establecer que la educación "pragmatista" y la "abierta," eran antagónicas. El resto de su argumentación 
se diluyó en la proposición de que el "pragmatismo" era una ideología capitalista, un instrumento para convertir la educación en algo que fuese útil para la clase capitalista; como "pensamiento idealista subjetivo de la educación" era claramente la antítesis de la ideología de la Revolución Cultural." Sus argumentos serían invertidos tras el final de la Revolución Cultural y el inicio del aperturismo político chino.

\section{Reabriendo la botella: el regreso de Dewey a China}

El final de la Revolución Cultural no llegó a ver el restablecimiento de Dewey. Este tuvo que esperar hasta el comienzo de la década de 1980. Mientras tanto, los artículos sobre Dewey revelaban una curiosa alianza entre éste y la Banda de los Cuatro: ¡La Banda de los Cuatro era pragmatista! Debido al uso cada vez más escurridizo del término "pragmatismo," la Banda de los Cuatro podía denominarse pragmatista porque sólo hacían lo que resultase ser útil para hacerse con el poder -y todo lo que para ellos fuese útil era "verdad." 67 "Pragmatista" se había convertido claramente en algo parecido a una blasfemia en los años 1970, y a veces se utilizaba como sinónimo de "fascista." Así mismo, la idea de la conspiración o intriga se extendió hasta incluir a la Banda de los Cuatro: fueron acusados de reprimir a la clase trabajadora y de impedirles obtener el conocimiento que les hubiese ayudado a derrocar el sistema capitalista; manteniendo a los estudiantes alejados del verdadero conocimiento, los hicieron a "actuar a ciegas." ${ }^{6}$

La Banda de los Cuatro provocó el caos que los críticos de Dewey de los años 1950 y 1960 ya habían anticipado en el caso de que su pedagogía centrada en el niño se pusiese en práctica: la des-centralización en el profesor llevó a vulgares faltas de respeto, la auto-organización de los estudiantes llevó al anarquismo sin límites, y la idealización de la ignorancia llevó a la ausencia total de cualquier conocimiento sistemático. Incluso se repitió el argumento del "biologicismo:" al igual que Dewey defendía que el niño tenía "talentos innatos," la Banda de los Cuatro sostuvo que el joven era "revolucionario por naturaleza” y sólo necesitaba crecer -sin profesor alguno. Escritores de los años 1970 se mostraron profundamente impresionados por la inversión radical de los valores educativos que trajo consigo la Revolución Cultural, que dio lugar a ataques salvajes contra el profesorado y contra todo "conocimiento libresco." Fang Yan narró, en una nueva comparación entre Dewey y la Banda de los Cuatro, ${ }^{69}$ un acontecimiento particularmente representativo y execrable en el que el estudiante Zhang Tiesheng, quien en 1973 se presentó al examen de ingreso en la universidad y que supuestamente entregó un "black paper," fue utilizado por la Banda de los Cuatro para radicalizar aún más su asalto a los "conocimientos tradicionales." En su culto a la ignorancia, los analfabetos se compararon con los bueyes salvajes valientes y fuertes. (Los estudiantes debían dejarse crecer los cuernos en la cabeza y espinas en el cuerpo para luchar heroicamente contra los capitalistas.)

A través de su condena de una política y una ideología dirigidas en contra de la adquisición de conocimientos sistemáticos, los escritores de la década de 1970 volvieron a introducir el concepto de "especialista" en el debate -el requisito previo para la modernización de China. Al situar a los Revolucionarios Culturales y los comunistas 
utópicos al mismo nivel que Dewey -cuya postura anticientificista e inmoral eran una verdad bien establecida-, los escritores podrían desvelar la vulgaridad y la falta de sentido de la actitud hostil hacia el conocimiento. ${ }^{70}$ Fang Yan, en un tercer artículo, citando a Chen Boda (1905-1989), un antiguo ideólogo comunista, decía: "No necesita tanta teoría, usted no puede comer, ni beber [teorizando; BS], si se puede conducir un tractor, es suficiente," antes de subrayar la importancia de conseguir "la educación roja y especializada," a fin de alcanzar los objetivos de las "cuatro modernizaciones." Por último, jugando la carta patriótica, Fang Yan acusó a la Banda de los Cuatro de intentar, con su política anti-modernista, arrastrar de nuevo a China al colonialismo. ${ }^{71}$

Empezando con el aperturismo político de Deng Xiaoping, los artículos sobre Dewey fueron perdiendo su emotiva polémica. Mientras algunos autores seguían ocupándose de la comparación entre Dewey y la Banda de los Cuatro, otros comenzaron -lentamente y con precaución- a mencionar su pensamiento educativo. Esto también se debía al hecho de que se empezase a releer y a revisar los trabajos de investigadores occidentales en revistas como Filosofia Mundial (Shijie Zhexue), Ciencias Sociales Avanzadas (shehui Kexue Zhanxian), o Investigación de Educación Comparada (Bijiao jiaoyu Yanjiu). Sin embargo, desde un punto de vista político-ideológico, las similitudes entre el marxismo y deweynismo se negaron con vehemencia. ${ }^{72}$ El lento restablecimiento de Dewey tuvo lugar de manera indirecta: a través de una nueva apreciación del pensamiento educativo de Tao Xingzhi y de sus contribuciones a China, se revisó a Dewey como de pasada. Así, Tao actuó como mediador de Dewey por segunda vez -aunque ambos fueron ignorados en la década de $1980 .{ }^{73}$ Quienes escribieron sobre Tao Xingzhi a principios de 1980 dejaron claro que Tao no era ni capitalista, ni pragmatista ni deweyano; sin embargo, la revisión del legado de Tao preparó el terreno para una recepción más positiva del pensamiento de Dewey, puesto que la discusión del pensamiento educativo de Tao iniciaba un debate en torno a la democracia y la ciencia.

En un artículo de 1981, Chen Tao Hancai describió cómo Tao "aceptó críticamente los contenidos de la teoría educativa de Dewey que tenían sentido, pero desechó el resto," y "a partir de las necesidades de las masas populares de nuestro país, presentó los contenidos educativos innovadores." ${ }^{74} \mathrm{El}$ artículo de Chen sentó las bases para la discusión sobre Dewey de la década de 1980; se abordaron casi todos los grandes temas (a excepción de la formación profesional) que iban a caracterizar el discurso de esos años: la democracia y las "masas," la ciencia y la innovación, el marxismo y la revolución, y el "carácter chino" de la modernización y la reforma de China. Hasta 1982, las posibles contribuciones de Dewey a este discurso se reconocieron con reservas; mientras se aceptaba que podían haberse derivado algunos efectos secundarios positivos de la teoría de Dewey, el núcleo de su pensamiento aún se rechazaba bajo el argumento de que era "capitalista" y estaba lleno de "idealismo subjetivo." El establecimiento de estas características negativas cambió claramente a partir de 1983: ya no dominaban el argumento general de los artículos, sino que se situaban al comienzo o al final del texto, para contentar a la censura y mantenerse a salvo de ella. Este cambio también podría haberse debido al hecho de que en 1982, la 
Asociación China de Investigación sobre la Historia de la Educación (Zhongguo Jiaoyushi Yanjiuhui) presentó un monográfico sobre Dewey en su conferencia anual. ${ }^{75}$

\subsection{La democracia y las masas}

La "Democracia" es un tema propio de los programas de los años 1980, aunque menos vinculado a los derechos de cada individuo que al bienestar de las masas. Los autores recordaban a los lectores que para Xingzhi Tao, discípulo de Dewey, la democracia significaba "penicilina para la política y vitaminas para la mente." ${ }^{76} \mathrm{Al}$ mismo tiempo, ser demócrata quería decir ser un hombre del pueblo, como Tao: "Cuando buscamos el conocimiento, ¿qué es lo que estamos haciendo? ¿Convertirnos en funcionarios y hacernos ricos? ¿Para que nuestro círculo más cercano se beneficie? ¡No! ... El camino del gran aprendizaje es: iluminar las virtudes de la gente, estando cerca de las masas, hasta que el pueblo [logre; BS] su felicidad." 77 Debido al aperturismo político chino, este tipo de actitud se consideró esencial para el logro de las Cuatro Modernizaciones. Se consideró adecuado al educador republicano Tao Xingzhi para encarnar el nuevo programa de modernización del país, incorporando al mismo tiempo a las masas. De pronto, se convirtió en una figura clave de la década de 1980. El hecho de que Dewey, con su programa educativo, se dirigiese a las masas (y no sólo a un pequeño grupo de elegidos) y que quisiese iluminarlos, hizo que ahora se le reconsiderase positivamente. Un aspecto más en el resurgimiento del interés por Dewey, fue su acentuación de la "vida real" y de lo que era verdaderamente relevante para la gente.

Sin embargo, también se vieron los inconvenientes de la teoría de Dewey, que Tao Xingzhi, como discípulo chino suyo aunque no imitador, logró superar: la "democratización exagerada." Con la experiencia de la Revolución Cultural aún muy reciente en sus mentes, los investigadores chinos eran más que prudentes cuando se trataba de "dejar que el niño haga lo que quiera hacer." En cambio, se consideró que Tao Xingzhi había defendido una noción de "disciplina" que no existía en el pensamiento de Dewey -pero que resultaba esencial para la "educación socialista." 78 Además, se consideraba que la concepción que Tao tenía de las masas era diferente de la de Dewey, lo cual se hizo evidente a través de cómo aquél alteró el lema deweyano de "la educación es vida" y lo convirtió en "la vida es educación." Se presentó a Tao como alguien realmente involucrado en la superación de las dificultades y penurias de la clase trabajadora, mientras se decía que el objetivo principal de Dewey era hacer que la clase trabajadora se adaptase a las dificultades y penurias existentes. Se informó de que Tao había tachado la visión educativa de Dewey de “jaula de pájaro,” donde se colocaban unas pocas ramas para simular la naturaleza -los estudiantes sólo tenían contacto con una "sociedad falsa" en la escuela. ${ }^{79}$

\subsection{Ciencia e innovación}

Además de la democracia, la ciencia era el otro eslogan de los años 1980 -lo que hizo de este período, también a los ojos de muchos intelectuales, sorprendentemente parecido a la época del Movimiento del Cuatro de Mayo, en el que el "Sr. Ciencia" y 
la "Sra. Democracia" tomaron las riendas. ¿Cómo podría volver a conectarse a Dewey con la idea de ciencia -sólo unos pocos años después de haber sido definido como la antítesis de la ciencia y ser considerado, incluso, como una amenaza para la adquisición de conocimiento científico sistemático? Una vez más, la transformación se produjo vía Tao Xingzhi, quien veía "el mundo actual [como] un mundo de ciencia, la nación china al completo debe recibir el bautismo de la ciencia..." ${ }^{80}$ Tao también trajo de nuevo a colación la importancia de la educación especializada y el conocimiento de los libros: mientras que Dewey, en la década de 1980, seguía siendo descrito como alguien que desecha los conocimientos librescos, Tao sólo quería acabar con la "aburridísima" actitud hacia el conocimiento de los libros. En lugar de "leer libros aburridos, leer libros de una manera aburrida, y leer libros para aburrirse," los estudiantes deberían "leer libros vivos, leer libros de manera vivaz, y leer libros para vivir." 81

Sin embargo, a partir de 1982 las contribuciones de Dewey a la ciencia de la educación pueden reconocerse en sus propios términos, sin que Tao Xingzhi funcione como mediador. Ahora Dewey puede denominarse de nuevo "progresista:" aunque hubiese algunos defectos en su teoría, en su pensamiento, "en el gran camino del desarrollo espiritual y civilizatorio de la humanidad, tuvo algunos efectos progresivos en medio del proceso de transformación de la teoría educativa." ${ }^{82}$ Los autores comenzaron a conceder gran importancia al enfoque "basado en la experiencia" de Dewey, ya que entendieron que ese acercamiento se adaptaba perfectamente a los tiempos de la modernización. Sin embargo, el antes mencionado Wang Tianyi seguía desconfiando del excesivo énfasis en la individualidad del niño, haciéndose eco de sus colegas de los años 1950 y 1960 así como de las preocupaciones de la derecha tras la Revolución Cultural:

Este énfasis [sobre la individualidad y la libertad del niño; BS] no sólo da libertad a los niños, sino que también los libera demasiado [...] Una consecuencia aún más grave de este énfasis es la que trae el caos al habitual orden de las escuelas, de modo que los profesores ya no son capaces de enseñar, los estudiantes ya no son capaces de estudiar, y las reglas que una escuela debe tener se disuelven en el caos [.... Éste es el carácter real de la "revolución” o "transformación" de Dewey en materia educativa. ${ }^{83}$

Sin embargo, sus temores se disiparon rápidamente en los artículos siguientes. Qiao Youhua sostuvo que la postura supuestamente anti-científica de Dewey era un malentendido, así como también lo era su anunciada disolución de todo tipo de disciplina en falta de respeto y caos. ${ }^{84}$ La imagen de Dewey se transformó gradualmente en la figura de alguien que respetaba mucho la ciencia pero que quería conectarla con la experiencia individual del estudiante. ${ }^{85}$ Finalmente, Dewey sirvió incluso como garantía de orden y estabilidad, y como una advertencia para no irse a los extremos (como había ocurrido durante la Revolución Cultural). Dewey, según lo que estos autores afirmaron, no había aniquilado completamente la tradición y, lo que es aún más importante, había ofrecido a cambio una filosofía nueva. Para los educadores de los 
años 1980, esto dio lugar a una acuciante pregunta: ¿qué tipo de filosofía necesitaba la nueva educación china? ${ }^{36}$

La idea de la innovación estaba estrechamente asociada a cómo los educadores chinos interpretaban ahora el acercamiento de Dewey a la ciencia. Teniendo en cuenta que la "innovación" iba a ser uno de los principales pilares de la modernización china, Dewey parecía especialmente prometedor en esta búsqueda. Anteriormente demonizadas o al menos ridiculizadas, las aportaciones de Dewey a la psicología del niño de pronto comenzaron a llamar la atención. La noción de "talento" (rencai) regresó -no como una entidad política, sino como un potencial innato que puede desarrollarse mediante el tipo de educación adecuado. A principios de los años 1980, la idea de "talento innato" sólo fue aceptada con cautela y compensada con la idea de formación. Así, Wang Tianyi anunció estar en contra de descuidar la formación por poner un excesivo énfasis en el desarrollo individual: "habrá ocho o nueve de cada diez que no serán capaces de convertirse en talentos útiles. Un ser humano necesita definitivamente pasar por la educación, y también tiene que pasar por una formación paciente y meticulosa." De lo contrario, la humanidad tendría que volver a la "vivir en cuevas y en el desierto." ${ }^{87}$ El final de la década de 1980, en vísperas de la Masacre de Tiananmen, fue testigo de una plena adopción del énfasis de Dewey sobre la individualidad y la creatividad; no había otro camino para convertirse en un país innovador:

Los análisis y críticas acerca de que a Dewey se le presentase con reservas en la teoría de la educación pasada y la por entonces práctica educativa occidental ¿ofrecen argumentos que la educación escolar actual de nuestro país podría entrar a considerar o sacar lecciones de ellos? Creo que sí lo hacen. Recuerden cómo el compañero Liu Fonian señaló una vez con respecto a los exámenes de postgrado que había unos pocos que memorizaban mecánicamente y aprendían de memoria. Aprobar los exámenes de la escuela media, de la universidad, incluso de los programas de postgrado, todo está basado en la memorización mecánica y el aprendizaje memorístico. Estas personas nunca han prestado atención a la formación de su propia capacidad de pensamiento creativo, nunca han querido aprender una verdadera destreza y el conocimiento genuinamente práctico [...] En su futuro trabajo, inevitablemente enseñarán convencionalismos a los estudiantes, ¿cómo podemos, con este método, formar el carácter activo de los alumnos, desarrollar sus habilidades cognitivas? ${ }^{88}$

\subsection{Marxismo y revolución}

Curiosamente, el autor que acabamos de citar cerraba su artículo con un párrafo sobre Marx -aunque no, como por lo general había sido, como una especie de corrección o complemento a Dewey, sino para subrayar la importancia del pensamiento humano. Esto es sintomático de cómo se pulió la imagen del marxismo para poder adaptarse a los nuevos tiempos. Pero ¿cómo se reconciliaron Marx y la idea de revolución con el Dewey capitalista y estadounidense, que estuvo abiertamente en contra de la revolución como un medio para cambiar la sociedad? 
Una vez más, Tao Xingzhi actúa como mediador en la fase inicial del restablecimiento. A través de él, los pensamientos de Dewey podrían evolucionar hacia un verdadero pensamiento dialéctico, uniendo la enseñanza, el aprendizaje y la acción. Por otra parte, Tao reconoció rápidamente la necesidad de la revolución, y de una educación al servicio de ésta. Así, fue visto como quien había conseguido transformar el discurso de la "educación para la supervivencia" (jiaoyu jiuguo) y del que se consideraba que Dewey formaba parte, en el discurso de la "revolución para la supervivencia” (geming jiuguo). ${ }^{89}$ Aparte de eso, la noción de "revolución" (y su necesidad) fue desapareciendo. El marxismo moderno podía actuar cada vez más sin necesidad de la revolución, y en este proceso Dewey fue siendo cada vez menos capitalista: ¡el sistema escolar tradicional contra el que Dewey hizo cruzada había sido después de todo un sistema de enseñanza capitalista! ${ }^{90}$ Asimismo, la resistencia de Dewey a la segregación de los estudiantes a través de la educación mediante la provisión a un grupo de una formación que "prepararse para la vida ociosa," y al otro grupo de una que "prepararse para la vida de trabajo productivo," era percibida ahora como anti-capitalista. ${ }^{91}$

Otros diferenciaron entre un Dewey teórico y un Dewey práctico: mientras que su actitud política estaba "equivocada en la teoría," su enfoque produjo resultados útiles en la práctica. ${ }^{92}$ Pero incluso los filósofos, que por su naturaleza, estaban más involucrados en la teoría de Dewey que sus homólogos en el ámbito educativo, empezaron a ver buenos argumentos en Dewey. Dewey fue elogiado por su método dialéctico, y en su crítica al marxismo se entendió que se dirigía sobre todo a los marxistas "falsos" (así como los marxistas "buenos" se habían equivocado), quienes habían propuesto teorías de determinismo económico. Los autores también reconocieron que en las sociedades socialistas, podían haberse "dado" algunos elementos "feudales" que eran incluso peores que el capitalismo (como el estalinismo), y Dewey había hecho bien en criticar estos “elementos." ${ }^{33}$ Dicho así, Dewey ya no era una amenaza para el socialismo como tal, sino que simplemente lo era -y con razón- para sus deformaciones.

\subsection{Modernización "à la chinoise"}

También se redescubrió a Dewey en el terreno de su -a ojos de los chinos- equilibrado punto de vista sobre la sociedad y el individuo. Mientras que sólo unos años antes se había ganado la fama de representar el individualismo y egoísmo extremos, en los años 1980 sufrió una sorprendente metamorfosis y emergió como el prudente término medio entre el concepto rousseauniano de una educación "demasiado individualista” y los programas nacionalistas alemanes o japoneses que tenían por objeto someter a los futuros ciudadanos completamente a los intereses del Estado. ${ }^{94}$ Dewey fue visto como la conciliación de la aparente contradicción y tensión de ambos, a través de una educación que sirviese a los intereses del individuo y de la sociedad. En la década de 1980 -al igual que hoy en día- esto se percibía como uno de los mayores retos: cómo individualizar la sociedad sin desocializar al individuo. Dewey parecía ofrecer respuestas útiles en este sentido. Un tono de reconciliación similar comenzó a invadir la simultaneidad de los conocimientos "antiguos" y los "nuevos:" ahora se entendía a Dewey como aquel que había hallado la manera de transmitir el 
conocimiento acumulado a lo largo de la historia de la humanidad uniéndolo a las experiencias nuevas e individuales. ${ }^{95}$

Por último, los autores chinos se identificaron cada vez más con Dewey y la situación a la cual se había enfrentado a lo largo su vida: una situación "en la que nos estamos enfrentando a una nueva ruptura a través de la industria, la economía y la tecnología." ${ }^{6}$ La situación histórica de Dewey no sólo se describía como una etapa en la que el capitalismo se encontraba al borde del colapso, sino como una etapa de suma importancia para el desarrollo tecnológico y la innovación, como una época de grandes desafíos, ante los cuales la educación tenía que reaccionar y ajustarse. El retrato de la educación deweyana también se reajustó con éxito, pasando de ser una educación "sin fines definidos" (y por lo tanto "inútil”) a uno de los instrumentos más poderosos e influyentes para cambiar la sociedad. ${ }^{97}$

\subsection{Formación professional}

A mediados de los años 1980 una parte más del pensamiento educativo de Dewey se recuperó y reactivó en China: sus ideas sobre la formación profesional. En las fases más ideologizadas de la educación china, apenas había espacio para la formación profesional: si cada trabajador podía convertirse en médico y cada agricultor en profesor, nadie tenía la necesidad de acudir a la formación profesional. Además, como se ha señalado anteriormente, la formación profesional era, por un lado, fuertemente criticada como un instrumento para apaciguar a las masas y reproducir así las reglas del sistema capitalista: contentando a los trabajadores, éstos nunca se emanciparían. Por otro lado, la interpretación "especializada" de la formación profesional como una "formación técnica" era considerada por muchos como demasiado restringida en su definición y no adaptada al tipo de educación socialista. Mientras que en los años 1950 y 1960 Dewey había sido claramente marcado por usar la estrategia capitalista del "apaciguamiento" en la formación profesional, los autores de mediados de la década de 1980 y en adelante revisaron la concepción holística de la formación profesional deweyana, la cual se consideraba que impregnaba toda su perspectiva educativa. Y lo que es más, con la ayuda de Dewey, la formación profesional podría representar mucho más que una formación en una tecnología o habilidad especial. Ahora era la vida misma, y una herramienta para ayudar a que cada individuo se desarrollase al máximo.

Este nuevo tipo de formación profesional de inspiración deweyana se dirigía a dos grupos: los trabajadores y los niños. Como señaló Wang Peixiong, los nuevos tiempos necesitaban de una educación que pudiese "hacer que los trabajadores fuesen conscientes de la base científica y social de su vocación, así como del significado de esa vocación." ${ }^{98} \mathrm{La}$ formación profesional se entendía ahora como parte del proceso de desarrollo de cada individuo y no como el homólogo técnico de la educación general. Con respecto a la educación de los niños, la formación profesional consistía en alcanzar las funciones morales. Refiriéndose a las nociones de Dewey de "empatía social" y "sentido de responsabilidad," los autores instaron a dedicarse a la formación profesional no sólo por razones económicas, sino para desarrollar la competencia 
social del niño y hacerle comprender las profesiones -y, en consecuencia, la vida realde una manera integral y científica. En esta línea de argumentación, ya no se mencionaba que los métodos de Dewey condujeron al caos y la anarquía. Por el contrario, su pensamiento educativo fue visto como capaz de proporcionar directrices a una sociedad que, debido al cambio en la concepción del marxismo, estaba perdiendo progresivamente su base ideológica. ${ }^{99}$

Según los artículos publicados, el final de los años 1980 asistió a una oleada de Dewey, seguida del silencio en la segunda mitad de 1989, justo después de la supresión del movimiento democrático. Como Beate Geist dice:

El 4 de junio trajo consigo el (pluralista) debate sobre la cultura que tuvo lugar en los años 1980 hasta su fin. A ello le siguió una prescriptiva recolección de la larga y gloriosa historia y cultura chinas, con el objetivo claramente político de contrarrestar la lucha contra el tradicionalismo y el nihilismo nacional, histórico y cultural de los años anteriores. Se hace hincapié en la "difusión de la excelente cultura nacional" (hongyang minzu youxiu wenhua). La intención era fortalecer la confianza y la unidad nacionales -y la estabilidad social. ${ }^{100}$

En octubre de 1989, Confucio fue repuesto en su trono, el viejo maestro de las relaciones sociales y del individuo con el Estado. Los intelectuales estaban ocupados con la "búsqueda de los valores educativos que acentúen por igual las necesidades sociales y de desarrollo individual," como rezaba el título de un artículo de $1990 .{ }^{101}$ Este artículo volvía a caer en los argumentos anteriores en los cuales Estados Unidos era utilizado como ejemplo disuasorio de lo que es un modelo educativo del "auto-servicio," que daba lugar a un individualismo egoísta por parte de los estudiantes. La sociedad socialista, en cambio, se presentaba como el acuerdo ideal donde se reconciliaban los intereses individuales y sociales. La educación socialista consistía en "ajustarse” a las necesidades de la sociedad, pero también en prestar la máxima atención al desarrollo de cada persona. Otros objetivos fueron los de combinar lo mejor de la cultura china y la occidental, revivir la cultura tradicional, y construir una nueva cultura que se correspondiese con la estructura política y económica actual y, por último, promover la "evolución de la raza y el progreso de la sociedad." 102

Las críticas hacia Dewey no tardaron mucho tiempo en volver. Pronto se descubrió a Dewey como una manera de salir del triángulo de las Bermudas compuesto por el Estado, la sociedad y el individuo. Dewey fue visto como una solución al conflicto entre el individuo y la sociedad a través de su idea de que el uno no podría existir sin la otra. Debía enfatizarse el desarrollo individual, pero por el bien de la sociedad, que a su vez sólo podría prosperar si las personas se desarrollaban plenamente. Esta “armonización” de los intereses individuales y sociales se lograría a través de la escuela. Basándose en los escritos de Dewey sobre la escuela, ésta sería una pequeña sociedad cooperativa en sí misma. Frente a las primeras críticas acerca de que la escuela deweyana seguía estando demasiado alejada de la realidad, la escuela moderna no debía confundirse con la sociedad, sino convertirse en "un mejor tipo de sociedad." 103 En una jugada limpia que terminó de rematar el argumento, ¡la sociedad ideal de 
Dewey - no siendo ya objeto de crítica- sólo se consideró factible dentro el sistema comunista!

Iniciándose también la década de 1990, hubo un resurgimiento del interés en la República China. Tanto la China republicana como la China de los años 1990 se entrcruzaron. Los (históricos) temas conectados con Dewey que se abordaron dentro de esta línea de investigación pueden asociarse fácilmente con preocupaciones más actuales. Basándose en citas de Dewey, los autores relataron casos de resistencia frente a una "copia" excesiva de lo occidental y contra la supresión de la tradición. También recordaron el énfasis de Dewey en la existencia de un Estado fuerte que debía encomendar los asuntos de interés público (como el sistema de ferrocarril) en manos privadas. ${ }^{104}$ Más recientemente, la investigación sobre la República China ha establecido que las diferencias ideológicas entre Dewey, Mao y Marx fueron mucho más permeables: estos autores hablan de una influencia marxista en Dewey ${ }^{105}$ y de una influencia deweyana sobre Mao. ${ }^{106}$ En un momento en que cada vez menos gente está interesada en el marxismo, el descubrimiento de que la filosofía más representativa de los Estados Unidos, el pragmatismo, fuese originariamente una derivación del marxismo no hace más que incrementar el valor de esta ideología: "Esto expresa [...] la fuerza que posee el marxismo, y nos hace ser aún más decididos a la hora de utilizar el marxismo como la ideología que nos lidera y de ser consecuentes en el camino del socialismo de carácter chino.” 107

\section{Dewey en los actuales debates chinos -Una panorámica}

En este último apartado, voy a esbozar algunos de los temas que han estado dominando últimamente los debates chinos sobre Dewey. La atención a la individualidad, la creatividad y el talento aún ocupan un lugar destacado en la discusión ${ }^{108}$ y se han integrado en el debate sobre la "calidad educativa" (suzhi jiaoyu). La "calidad educativa” surgió en los años 1990 como una educación más integral y en oposición a la "educación orientada al examen" (yingshi jiaoyu); ${ }^{109}$ y fue concretándose en los programas educativos que se pusieron en marcha a principios del nuevo milenio. Se considera que Dewey y la "calidad educativa" comparten la misma visión sobre los objetivos, el currículo y los métodos educativos. La insistencia de Dewey en que "la educación es vida" y su énfasis en la experiencia y el "desarrollo activo" se consideran ampliamente compatibles con la perspectiva de la "calidad educativa." Ambas tienen como objetivo "elevar la calidad de los ciudadanos" y "hacer que esta nueva generación de ciudadanos se adapte aún mejor a la vida social cambiante y, lo que es todavía más importante, que creen una sociedad mejor y más progresista." 110

En consecuencia, a Dewey se le utiliza para justificar las reformas educativas que debieran alejarnos de la orientación hacia el examen, del conocimiento "inútil” y del tradicional estilo "militarista" de la educación. ${ }^{111}$ Cierto número de autores reformistas ven el conflicto entre la educación tradicional y la calidad educativa contenido en los dos educadores Herbart y Dewey: la perspectiva de Herbart sobre la educación se reflejaría en el estilo de enseñanza tradicional chino, mientras que el pensamiento educativo de Dewey se encontraría en el corazón de la actual reforma. Teniendo en 
cuenta que el principal papel que Dewey concede al profesor moderno supone un gran reto para el proceso de enseñanza actual, Zhou Hailing aboga por un enfoque dialéctico: "Por lo tanto debemos aprender un conocimiento fiable y útil, y buscar el conocimiento que aún desconocemos, absorber lo que ya tenemos y fomentar la exploración de caminos desconocidos." 112 Sin embargo, la mayoría de los autores aboga por "utilizar" a Dewey en la reforma actual de la educación, lo que hace cada vez más borroso al propio Dewey. Algunos autores se quejan de que en realidad Dewey planteó "exigencias de una naturaleza muy general, y que no proporcionaban un modelo claro y sencillo con el que trabajar." 113 Otros artículos lo interpretan abiertamente como algo extraño, por ejemplo cuando se anima a los estudiantes a desarrollar un "pensamiento independiente" y su "personalidad," pero al mismo tiempo, a seguir "la cosmovisión correcta acerca de la vida humana y de los valores." 114

Probablemente sea debido a esta especie de mantra que insiste en la "individualidad," por lo que se advierta contra una "aplicación" demasiado mecánica del pensamiento de Dewey a la "calidad educativa." Como Zhu Guohui y Luo Yaocheng recuerdan a sus lectores, un individuo bien situado -el objetivo de la "calidad educativa" - no puede lograrse sin una verdadera individualidad:

Durante mucho tiempo, la educación superior de nuestro país ha tenido como objetivo formar para la adaptación a la sociedad, la función principal de la educación era promover la socialización del individuo. La llegada silenciosa de la economía del conocimiento necesita urgentemente que la educación superior forme a un gran número de talentos humanos innovadores, pero la clave para la formación del talento humano innovador radica en el desarrollo de la individualidad de la persona, lo cual ha provocado un nuevo desafío para la educación superior de nuestro país, que ha estado tradicionalmente "orientada hacia la sociedad." 115

Así, no sólo se utiliza a Dewey para legitimar los nuevos programas educativos tales como los de la "calidad educativa," sino también para evaluar críticamente si estos programas se están llevando realmente a cabo -o si sólo sirven de estandartes retóricos superficiales.

La concepción de la "calidad educativa" está muy unida a una discusión más amplia sobre lo que debería ser la base moral y ética de la sociedad de hoy. Los artículos de la última década siguen reflejando la búsqueda del adecuado equilibrio entre la individualización y la socialización. Varios autores utilizan comparaciones históricas para subrayar la importancia compartida de la individualidad y la sociabilidad. Mientras que Confucio ${ }^{116}$ y el mencionado filósofo Liang Shuming ${ }^{117}$ se emplean -junto con Dewey- como ejemplos más tradicionales que pueden propagar la moralización de la sociedad, Mao Zedong sirve (una vez más, junto con Dewey) como una legitimación más reciente de que la "moral" es tan importante como el conocimiento. Actualmente Dewey es visto como alguien que consideró la "educación moral" como el medio de mantener la estabilidad social y de promover la economía. Así, la anterior acusación de que Dewey utilizaba la educación para manipular y atontar a la clase 
obrera se ha convertido en una revisión completa de su presunto uso instrumental de la educación. ${ }^{118}$

Casi inevitablemente, estos grupos discursivos sobre la "calidad educativa," el "individuo y la sociedad" y la "educación moral" se mezclan en el proyecto del actual presidente, $\mathrm{Hu}$ Jintao, de "construir una sociedad socialista armoniosa." ${ }^{119}$ La idea de Dewey de situar a la persona en el centro es vista como la "base para construir una sociedad armoniosa," 120 que no puede permitirse tener estudiantes que "no participan de las actividades más básicas de la sociedad" y por lo tanto tiene que poner más énfasis en la educación moral. ${ }^{121}$ También la formación profesional, continuando con la interpretación de Dewey que se había iniciado a mediados de los años 1980, es vista como un instrumento para llevar a cabo esta sociedad armoniosa -pero sólo si no se reduce a la capacitación técnica. La armonía se logra de dos maneras: en primer lugar, la formación profesional ayuda a encontrar la profesión adecuada, desde la cual se obtendría la satisfacción: "En general, la actividad laboral es el único medio de equilibrar los talentos específicos de una persona con su servicio a la sociedad." ${ }^{122}$ En segundo lugar, la transmisión de una ética profesional capacitaría a "personas armoniosas" y a "trabajadores de alta calidad," lo cual evitaría que fuesen "máquinas inútiles." 123 Evidentemente, "rojo" y "especialista" se han reconciliado para convertirse en la feliz pareja de la "armonía" y la "calidad."

Sin embargo, Dewey no sólo representa la ideología del Estado. Su autoridad moral en los círculos académicos chinos no se agota en las repetidas referencias a una "sociedad armoniosa." También es utilizado como un arma contra el "neoliberalismo" -como el verdadero representante de un liberalismo que no tiene nada que ver con el neo-liberalismo chino de la década de los salvajes años $1990 .{ }^{124}$ Se señala que Dewey nunca habría sacrificado la "igualdad" en aras de la "libertad" -como algunos defensores chinos del liberalismo harían hoy. La libertad no debe estar en posesión de una pequeña minoría (capitalista), sino del mayor número posible de personas, incluso si esto significase para el Estado restringir la libertad en algunas áreas bien definidas. Asimismo, otro artículo señala el énfasis de Dewey en la capacidad de dirigir el Estado -en contraste con las opiniones de "antiguos" liberales como Locke. La democracia, según esta opinión, no era el medio de lograr el liberalismo pero sí la base que otorga legitimidad moral a la sociedad para que discuta sus necesidades e intereses. ${ }^{125}$

Resulta interesante el hecho de que Dewey haya sustituido a Marx en este debate -quien hubiese sido la referencia más natural en cualquier argumento contra el neoliberalismo, al menos desde un punto de vista occidental. Así, Dewey ha superado con éxito tres metamorfosis: de antítesis de la ciencia y el conocimiento se transformó en el emblema de la ciencia innovadora; su carácter profundamente inmoral, egoísta y capitalista se transformó en un símbolo de la (casi apolítica) armonización del individuo y la sociedad; y, por último, de víctima del criticismo marxista-socialista se ha convertido en uno de los actores políticos del gobierno estatal chino en su vis-a-vis con la China del neo-liberalismo. John Dewey -o más bien Duwei- realmente se ha convertido tanto en un "rojo" como en un "especialista." 


\section{Notas}

1. Barry Keenan, The Dewey Experiment in China. Educational Reform and Political Power in the Early Republic (Cambridge, Massachusetts \& London, England: Harvard University Press, 1977).

2. En este mismo sentido, Jessica Wang argumenta que la "[mayoría] de las críticas sobre Dewey eran malos entendidos;" ver Jessica Ching-Sze Wang, John Dewey in China. To Teach and to Learn (New York: State University of New York Press, 2007).

3. Wang mantiene un argumento similar en su subcapítulo "Whose Teaching? Or Hu’s Teaching?," ibid., 30-40.

4. Esto también lo señala Bong-Ki Kim, Das Problem der interkulturellen Kommunikation am Beispiel der Rezeption Deweys in China, Duisburg Working Papers on East Asian Studies no. 19 (Duisburg: Institute for East Asian Studies, Gerhard-MercatorUniversität GH Duisburg, 1999).

5. Ver James Scott Johnston, "Can Democratic Inquiry Be Exported? Dewey and the Globalization of Education," Democracy as Culture: Deweyan Pragmatism in a Globalizing World, ed. Sor-hoon Tan y John Whalen-Bridge (Albany, NY: SUNY Press, 2008), 69. Johnston cita a Dewey diciendo que "el problema es extraer las características deseables a partir de las formas de vida comunitaria que realmente existen, y utilizarlas para criticar las caracteristicas no deseadas y sugerir mejoras"; ibid., 76.

6. Citado en Keenan, The Dewey Experiment, 7.

7. En este movimiento patriótico-revolucionario, no sólo los intelectuales y los estudiantes protestaron contra la pérdida de soberanía nacional, sino también abogaron por una 'nueva cultura' que rechazaba la tradición confuciana y que debería ser accesible para todos, tanto en términos de la ideología y como del idioma.

8. Hu Shi fue un famoso escritor y una prominente figura del Movimiento del Cuatro de Mayo. Luchó a lo largo de su vida por una nueva cultura y lengua chinas que fuesen inteligibles para todos (en contraste con el chino clásico). Después de sus estudios en el Centro de Formación de Profesorado, fue profesor en la Universidad de Beijing (y más tarde rector).

9. Sobre este péndulo ideológico de la educación china, ver por ejemplo Chan Hoiman, "Modernity and Revolution in Chinese Education: Towards an Analytical Agenda of the Great Leap Forward and the Cultural Revolution," Education and Modernization. The Chinese Experience, ed. Ruth Hayhoe y Zhu Weizheng (Oxford: Pergamon Press, 1992), 73-99.

10. Ver por ejemplo Zhou Ye, “'Xin Jiaoyu' yu Zhongguo jiaoyu jindaihua [The Journal Progressive Education and the Modernization of Chinese Education]," Gaodeng Jiaoyu Yanjiu 26, 1 (2005): 83-84. Esto se afirma a pesar de que a finales de los años 1930, más de 5000 escuelas progresivas estaban en funcionamiento en los Estados Unidos. Agradezco a los evaluadores ciegos esta información.

11. Huang Yanpei, "Xuexiao jiaoyu caiyong shiyongzhuyi zhi shangque [Debate sobre el uso del pragmatismo en la educación escolar]," Jiaoyu Zazhi 5, 7 (1913) (supplement): $55-82$.

12. Li Hongzhang, "Jiangsu xunfu Li Hongzhang yuanhan [The Original Letter by Li Hongzhang, Provincial Governor of Jiangsu]" [1864], Chouban Yiwu Shimo: Tongzhichao [Chronicle of Barbarian Matters from the Beginning to the End [of the Qing-Dynasty]: TongzhiPeriod], vol. 5, section 25 (Taibei: Guofeng Chubanshe, 1963), (originally 4-12), 624. 
13. Hu Shi, Hu Shi Koushu Zichuan [Hu Shis Oral Autobiography], traducido por Tang Degang (Beijing: Huawen Chubanshe, 1989), 107-08. Esta es la traducción china de las gravaciones que recogen las memorias de $\mathrm{Hu}$ Shi y que se encuentran en la Columbia University.

14. Ver Xia Jun, "Duwei shiyongzhuyi lilun yu Zhongguo xiangcun jianshe yundong [La teoría pragmatista de Dewey y el movimiento para el desarrollo del campo en China]," Minguo Dang'an 3 (1998): 63-69, 63.

15. Ver Yuan Qing, "Duwei de Zhongguo zhi xing jiqi yingxiang [Dewey in China and His Influence]," Jindaishi Yanjiu 2 (2001): 149.

16. Yuan Qing denomina al nuevamente introducido 'pragmatismo' como una arma para los intelectuales chinos con la que lucha contra la educación tradicional; ver Yuan, "Duwei de Zhongguo zhi xing."

17. Sor-hoon Tan, "China's Pragmatist Experiment in Democracy: Hu Shih's Pragmatism and Dewey's Influence in China," Metaphilosophy 1/2 (2004): 51-52.

18. Citado en Y. C. Wang, "Intellectuals and Society in China 1860-1949," Comparative Studies in Society and History 3, 4 (1961): 410.

19. Ver por ejemplo Yang Fengyin, "Duwei he Luosu de Zhongguo jindaihua fang'an [Dewey's and Russell's Programs for China's Modernization]," Jianghuai Luntan 4 (2002): $68-72$.

20. Wang, John Dewey in China, 33 (ver n. 2). De acuerdo con Wang, Dewey estaba preocupado de ser 'utilizado': "Dewey sabía que lo que simbolizaba era más importante que lo que decía;” ibid., 39.

21. Keenan, The Dewey Experiment, 13 (ver n. 1).

22. Yuan, "Duwei de Zhongguo zhi xing," 139ss. (ver n. 15).

23. Zhou Ye, "Xin Jiaoyu' yu Zhongguo jiaoyu jindaihua [The Journal Progressive Education and the Modernization of Chinese Education]," Gaodeng Jiaoyu Yanjiu 26, 1 (2005): 87-92.

24. Yuan, "Duwei de Zhongguo zhi xing," 142; Zhang Liangcai, "Duwei lai Hua dui Zhongguo jiaoyu de yingxiang [The Influence of Dewey's Visit to China on Chinese Education]," Liaoning Gaodeng Jiaoyu Yanjiu 89, 6 (1996): 97-102.

25. Wang, John Dewey in China, 4 (see n. 2). Ver el capítulo de Jeremy Rappleye sobre Japón en este volumen.

26. Ver su octava carte en Carl Heinrich Becker in China. Reisebriefe des ehemaligen preußischen Kultusministers 1931/32, ed. Susanne Kuß, Berliner China-Studien/Quellen und Dokumente (Münster: LIT, 2004), 151.

27. Ni Zhuhua, "Xiaoxue gongyike jiaoxue he shebei de shangque [A Discussion of the Didactics and Facilities of Craftsmanship Instruction at Primary Schools], "Jiaoyu yu Zhiye 26 (1921): 1-6.

28. "Zhonghua Zhiye Xuexiao gaikuang [The General Situation of the Chinese Vocational School], “Jiaoyu yu Zhiye 20 (1920): 1-6.

29. Ver Keenan, The Dewey Experiment, 23. Mientras que Wang subraya las diferencias de opinión entre Dewey y Hu Shi, no presenta evidencias convincentes de que Dewey fuese capaz de obtener información de primera mano sin recurrir a sus amigos chinos, dado su limitado conocimiento del idioma y la sociedad chinos (como él mismo admitió). En general, el relato de Wang está impregnado de la convicción fundamental de que Dewey, como una 
manera de solidarizarse con el pueblo chino y su visión de futuro, comprendió la complejidad de su sociedad; ver el capítulo 4 en Wang, John Dewey in China (ver n. 2).

30. Keenan, The Dewey Experiment, 10.

31. Ver su séptima carta en Carl Heinrich Becker in China, 133 (ver n. 26).

32. Cf. Li Sanfu, "Shilun Duwei shiyongzhuyi jiaoyu sixiang yu Zhongguo jiaoyuxue kexuehua [A Tentative Discussion of Dewey's Pragmatist Educational Thought and the Scientification of Chinese Educational Science]," Yunnan Shifan Daxue Xuebao 4 (2001): 13-17.

33. Esto fue paralelo al descubrimiento de la mujer como un ser humano complete. No era coincidencia que Alice Dewey diese conferencias sobre los derechos de la mujer durante sus dos estancias en China.

34. Sun Jianjiang, "Duwei 'Ertong Benweilun' jiqi yu Zhongguo ertong wenxue de guanxi [Dewey's 'The Child as the Center' and Its Relationship with Chinese Children's Literature],"Yunnan Shehui Kexue (1995): 89-94.

35. Ni, "Xiaoxue gongyike jiaoxue" (ver n. 27). 24).

36. Ver por emeplo Zhang, "Duwei lai Hua dui Zhongguo jiaoyu de yingxiang" (ver $n$.

37. Huang Yanpei, "Zhiye jiaoyu [Formación profesional]," Jiaoyu Zazhi 13, 11 (1921): 61-63.

38. Wang Maozu, "Jiaoyu shang shiyongzhuyi zhi weizhi [The Position of Pragmatism in Education]," Jiaoyu Zazhi 12, 7 (1920): 4.

39. Ver Gong Chushu, "Zai tan xiaoxue kecheng zhong de zhiye zhidao [Reconsidering Vocational Guidance in the Primary School Curriculum]," Jiaoyu yu Zhiye 98 (1928): 521-26.

40. Sobre Japón, ver la contribución de Jeremy Rappleye’s en este volumen.

41. Gong, "Zai tan xiaoxue kecheng zhong de zhiye zhidao," 525.

42. Ver por ejemplo Zhuang Yu, "Lun jiaoyu fangzhen [Discussing the Principles of Education]," Jiaoyu Zazhi 4, 1 (1912): 1-11.

43. Wang, "Jiaoyu shang shiyongzhuyi zhi weizhi," 3.

44. Shu Xincheng, "Zhongxue zhiye zhidao de xianjue wenti [Preconditions for Vocational Guidance at the Middle School]," Jiaoyu Zazhi 17, 1 (1925): 1-6.

45. Zhao Yichen, "Shiyan xiaoxue huo fushu xiaoxue yinggai zenmeyang [What Should Laboratory Primary Schools or Attached Primary Schools Be Like]?," Jiaoyu Zazhi 21, 5 (1929): 1-7.

46. Ver Wang, John Dewey in China, 50-51 (see n. 2).

47. Jiang Qi, "Zhiye jiaoyu de benzhi zhi yanjiu [An Investigation of the Basic Character of Vocational Education]," Jiaoyu yu Zhiye 136 (1932): 241-67.

48. Lu Guoqi, "Lun Duwei dui Mao Zedong zaoqi jiaoyu sixiang de yingxiang [Discussing Dewey's Influence on the Early Educational Thought of Mao Zedong],"Xue Lilun 5 (2009): 136-37.

49. Qi Xingui, "Duwei dui Zhongguo de yingxiang: yi zhong pipanxing pingjia [Dewey's Influence on China: A Critical Evaluation]," Chongqing Shizhuan Xuebao (Shehui Kexueban) 3 (1997): 17. Hu Shi habría corridor la misma suerte si no llega a emigrar a Taiwan. 
50. Cao Fu, "Duwei pipan yinlun (shangpian) [An Introduction to the Criticism of Dewey (first part)]," Renmin Jiaoyu 1, 6 (1950): 21.

51. Cao Fu, "Duwei pipan yinlun (xiapian) [An Introduction to the Criticism of Dewey (last part)]," Renmin Jiaoyu 2, 1 (1950): 23.

52. Cao, "Duwei pipan yinlun (shangpian)," 22.

53. Che Wenbo, "Pipan Duwei shiyongzhuyi jiaoyuxue fan kexue fan geming de benzhi - Dui Duwei Mingri zhi Xuexiao yi shu de pipan [Criticizing the Anti-Science and Anti-Revolution Essence of Dewey’s Pragmatist Pedagogy]," Renwen Kexue Xuebao 2 (1956): $105-28$.

54. Ibid., 106.

55. Cao, "Duwei pipan yinlun (shangpian)," 26.

56. Cao, "Duwei pipan yinlun (xiapian)," 28.

57. Che, "Pipan Duwei shiyongzhuyi jiaoyuxue fan kexue fan geming de benzhi," 124.

58. Cao, "Duwei pipan yinlun (xiapian)," 29 (ver n. 56).

59. Ibid., 29.

60. Zhong Chengzhang, "Fandui shiyongzhuyi guandian [Against the Pragmatist Perspective]." Jianghuai Luntan 5 (1963): 16-22.

61. Cao, "Duwei pipan yinlun (shangpian)" (ver n. 55).

62. Che, "Pipan Duwei shiyongzhuyi jiaoyuxue fan kexue fan geming de benzhi," 119 (ver n. 57).

63. Ibid., 114.

64. Jin Yuelin, "Pipan shiyongzhuyizhe Duwei de shijieguan [Criticizing the World View of the Pragmatist Dewey]," Zhexue Yanjiu 2 (1955): 13.

65. Cao, "Duwei pipan yinlun (shangpian)," 28 (ver n. 50).

66. Juan Zhi, "Shei shuo kaimen banxue shi 'shiyongzhuyi' [Who Says that OpenDoor Education Is 'Pragmatism']?," Renmin Jiaoyu 11 (1974): 41-42.

67. Liang Si, “'Si Ren Bang' weishenme haipa pouxi shiyongzhuyi [Why is the 'Gang of Four' Afraid of Analyzing Pragmatism]?,” Renmin Jiaoyu 2 (1977): 5.

68. Fang Yan, ''Sirenbang' de 'xuesheng zhizhi' yu Duwei de 'ertong zhongxin' [The 'Students' Self-Organization' of the 'Gang of Four' and Dewey's 'Child as the Center']," Shandong Shifan Daxue Xuebao (Renwen Shehui Kexueban) Z1 (1977): 109-10.

69. Fang Yan, 'Sirenbang' de 'wenmang jia yeniu' yu Duwei de 'jiaoyu ji shenghuo' [The 'Gang of Four's 'Illiterates Are Wild Oxen' and Dewey's 'Education Is Life']," Shandong Shifan Daxue Xuebao (Renwen Shehui Kexueban) 6 (1977): 11-13.

70. Esto se vio reforzado por el hecho de que durante la Revolución Cultural el lenguaje se vulgarizó mucho. Sobre la demonización del discurso entre los años 1950 y 1970 , ver Michael Schoenhals, "Demonising Discourse in Mao Zedong's China: People vs NonPeople," Totalitarian Movements and Political Religions 8, 3-4 (2007): 465-82.

71. Fang Yan, “'Sirenbang' de 'renwu dai jiaoxue' yu Duwei de 'cong zuo zhong xue' [The 'Gang of Four's' 'Teaching Mission' and Dewey's 'Learning by Doing']," Shandong Shifan Daxue Xuebao (Renwen Shehui Kexueban) 4 (1977): 61-65. Las 'Cuatro Modernizaciones' fueron formuladas por Zhou Enlai en enero de 1975 en el Cuarto Congreso del Pueblo y fueron proclamadas oficialmente por Deng Xiaoping en 1978. Incluían la modernizaicón agrícola, industrial, tecnológica y militar. 
72. Ver por ejemplo el artículo de Xu Chongwen, que estaba indignado por el George Novack, a veterano teórico del Partido Socialista de los Trabajadores de Estados Unidos, que comparaba partes de la tradición pragmatista con el pensamiento marxista; Xu Chongwen, "Makesizhuyi he shiyongzhuyi: Duili haishi tiaohe? — Ping Nuofanke: 'Dui Duwei zhexue yi ge pingjia: shiyongzhuyi tong makesizhuyi de duili' [Marxism and Pragmatism: Opposition or Harmony? - Reviewing Novack: 'An Appraisal of John Dewey's Philosophy. Pragmatism Versus Marxism']," Guowai Shehui Kexue 2 (1978): 90-93. El título correcto del libro bibliográfico de Novack es el siguiente: George Novack, Pragmatism Versus Marxism. An Appraisal of John Dewey's Philosophy (New York: Pathfinder, 1975).

73. Hu Shi fue restablecido cierto tiempo más tarde, a principios de 1986, beneficiándaose quizá también de la progresiva positive re-evaluación del pensamiento de Dewey que estaba entonces en curso. Debido a su actitud decididamente liberal y a su emigración a Taiwan, los debates en la China continental tuvieron que ser lo suficientemente abiertos como para apreciar las contribuciones de $\mathrm{Hu}$.

74. Chen Hancai, "Qianxi Tao Xingzhi yu Duwei jiaoyu sixiang de benzhi qubie [A Shallow Analysis of the Qualitative Differences between the Educational Thought of Tao Xingzhi and Dewey]," Huanan Shifan Daxue Xuebao (Shehui Kexueban) 4 (1981): 44.

75. Pang Dan sitúa el punto de inflexión hacia una recepción más abierta del pensamiento de Dewey mucho más tarde, en 1987; esto no puede verificarse por los artículos que se publicaron con anterioridad a dicha fecha. Sin embargo, tiene razón en señalar que la atención académica sobre la recepción de Dewey era sobre la educación, no sobre la filosofía. A diferencia de sus colegas más pragmáticos en cuanto a la educación, los filósofos mantuvieron una actitud mucho más despreciativa hacia la filosofía de Dewey. Ver Pang Dan, "Jin shi nian lai wo guo xuejie Duwei sixiang yanjiu pingshu [A Critical Account of My Country's Scholars' Research of the Past Ten Years on Dewey's Thought]," Lilun Tantao 1 (2008): 53-57.

76. Chen, "Qianxi Tao Xingzhi yu Duwei jiaoyu sixiang de benzhi qubie," 44.

77. Tao Xingzhi, citado en ibid., 46-47.

78. Ibid., 44-45.

79. Ibid., 49-50.

80. Ibid., 45.

81. Ibid., 51.

82. Wang Tianyi, "Duwei jiaoyu sixiang tanjiu [Exploring Dewey's Educational Thought]," Beijing Shifan Daxue Xuebao (Shehui Kexueban) 3 (1982): 74.

83. Wang, "Duwei jiaoyu sixiang tanjiu," 83.

84. Qiao Youhua, "Guanyu Duwei shiyongzhuyi jiaoyu sixiang zai pingjia de ji ge wenti [Some Questions Concerning the Re-Evaluation of Dewey's Pragmatist Educational Thought]," Dongbei Shida Xuebao (Zhexue Shehui Kexueban) 3 (1983): 66-70.

85. Zhang Fakun, "Duwei 'Minzhuzhuyi yu Jiaoyu' zhong de pipan jicheng wenti [Critical Succeeding Questions in Dewey's 'Democracy and Education']," Jiaoyu Yanjiu yu Shiyan 2 (1984): 89-95.

86. Wang Peixiong, "Duwei jiaoyu gaigeguan fawei [Some Small Points about Dewey's View on Educational Reform]," Bijiao Jiaoyu Yanjiu 5 (1986): 44-49.

87. Wang, "Duwei jiaoyu sixiang tanjiu," 78-79.

88. Li Yaling, "Duwei lun fazhan xuesheng de siwei nengli [Dewey Discusses Developing the Students' Capability to Think]," Heilongjiang Gaojiao Yanjiu 1 (1988): 71. 
89. Chen, "Qianxi Tao Xingzhi yu Duwei jiaoyu sixiang de benzhi qubie." See also Liu Zhiju, "Tao Xingzhi jiaoyu sixiang bu tong yu Duwei sanlun [Three Points Where the Educational Thought of Tao Xingzhi Is Different from Dewey]," Anhui Shifan Daxuebao (Zhexue Shehui Kexueban) 4 (1981): 16-22, 29.

90. Wang, "Duwei jiaoyu sixiang tanjiu," 77. $85)$.

91. Zhang, "Duwei 'Minzhuzhuyi yu Jiaoyu' zhong de pipan jicheng wenti," 89 (ver n.

92. Qiao, "Guanyu Duwei shiyongzhuyi jiaoyu sixiang zai pingjia de ji ge wenti" (ver n. 84).

93. Sun Yuecai, "Duwei de duoyuanlun ziyouguan — Jian lun dui shiyongzhuyi de pingiia wenti [Dewey's View on Pluralistic Freedom - Discussing Critical Questions of Pragmatism]," Shehui Kexue 4 (1985): 52-54, 13.

94. Zhang, "Duwei 'Minzhuzhuyi yu Jiaoyu' zhong de pipan jicheng wenti."

95. Li Yaling, "Duwei lun fazhan xuesheng de siwei nengli" (ver n. 88).

96. Wang Peixiong, "Duwei zhiye jiaoyu sixiang shuping [Comments on Dewey's Vocational Education Thinking]," Bijiao Jiaoyu Yanjiu 5 (1984): 45.

97. Cf. Wang, "Duwei jiaoyu gaigeguan fawei," 45 (ver n. 86).

98. Wang, "Duwei zhiye jiaoyu sixiang shuping," 45.

99. Sin embargo, a lo que los profesores de la formación professional se oponían, era al llamamiento de Dewey para la abolición de la escuelas profesionales por completo. Diferenciarse del sistema escolar se consideró importante, especialmente después de la experiencia con la educación 'unificada' de la Revolución Cultural.

100. Beate Geist, Die Modernisierung der chinesischen Kultur: Kulturdebatte und kultureller Wandel im China der 80er Jahre (Hamburg: Institut für Asienkunde, 1996), 217.

101. Zhou Zhichao y Zhang Wenchao, "Xunqiu shehui xuyao yu ren de fazhan bingzhong de jiaoyu jiazhiguan [Searching for Educational Values that Put Equal Stress on Society's Needs and Individual Development]," Jiaoyu Pinglun 3 (1990): 28-29.

102. Ibid., 29.

103. Xu Bangguan, "Lun Duwei de jiaoyu jiazhi quxiang [Discussing Dewey’s Educational Value Orientation]," Jiaoyu Yanjiu yu Shiyan 2 (1990): 38.

104. Ver por ejemplo Yang Fengyin, "Duwei he Luosu de Zhongguo jindaihua fang'an" (see n. 19).

105. Huang Xiaoyan y Zhang Quanxin, "Makesizhuyi zhexue shi Duwei 'Minzhu gongtongti' de lilun laiyuan [Marxist Philosophy Is the Theoretical Source for Dewey's 'Democratic Community']," Shandong Shifan Daxue Xuebao (Renwen Shehui Kexueban) 1 (2009): 10-14.

106. Lu Guoqi, "Lun Duwei dui Mao Zedong zaoqi jiaoyu sixiang de yingxiang" (see n. 48).

107. Huang y Zhang, "Makesizhuyi zhexue shi Duwei 'Minzhu gongtongti' de lilun laiyuan," 13.

108. Ver por ejemplo Pei Changling, "Gexing yu chuangzaoxing — Duwei jiaoyu sixiang gei women de qishi [Individuality and Creativity - Insight from Dewey's Educational Thought]," Qinghai Shizhuan Xuebao (Shehui Kexue) 2 (2001): 86-87; or, on pre-school education: Wei Cai, "Duwei bixia de 'jingyan' dui youeryuan jiaoxue de 
qishi [Inspiration from Dewey's 'Experience' for Kindergarten Pedagogy]," Kejiao Wenhui (Zhongxunkan) 1 (2009): 124-25.

109. Sobre la 'calidad educativa', ver por ejemplo Belinda Dello-Iacovo, "Curriculum Reform and 'Quality Education' in China: An Overview," International Journal of Educational Development 29 (2009): 241-49.

110. Yu Kangping, "Duwei yu suzhi jiaoyu [Dewey y calidad educativa]," Wenjiao Ziliao 3 (2008): 122-24.

111. Song Zhirun y Chen Xiaoping, "Cong Duwei de jiaoyu sixiang kan woguo de jiaoyu gaige [On the Reform of Education in China from the Angle of Dewey's Thought of Education]," Chuangxin 4 (2009): 13-15; 31.

112. Zhou Hailing, "Cong renshilun jiaodu kan Heerbate yu Duwei jiaoyu sixiang de fenqi [Looking at the Differences in Herbart's and Dewey's Educational Thought from an Epistemological Perspective]," Huabei Shuili Shuidian Xueyuan Xuebao (Shekeban) 2 (2006): 33.

113. Xia Dongjie and Guo Xu, "Shilun Duwei de jiaocaiguan jiqi dui xin kecheng gaige de qishi [A Tentative Discussion of Dewey's View on Teaching Material and Its Inspiration for the New Curriculum Reform]," Nei Mengu Shifan Daxue Xuebao (Jiaoyu Kexueban) 8 (2007): 63.

114. Yu Jie, "Duwei deyu sixiang dui wo guo xiandai deyu de qishi [An Inspiration from Dewey's Moral Education Thought for My Country's Present Moral Education]," Kejiao Wenhui 7 (2008): 279.

115. Zhu Guohui y Luo Yaocheng, “Duwei de 'Jiaoyu wu mudi lun' pingxi — jian tan woguo gaodeng jiaoyu mudi ying chuli hao de ji dui guanxi [A Critical Analysis of Dewey's 'Theory of Aimless Education' - Discussing Some Relationships with How to Deal Well with Our Country's Higher Education Aims]," Jilin Gongxueyuan Xuebao 4 (2001): 8.

116. Zhang Wu, "Xunzhao xinyang de shuguang — Kongzi yu Duwei yishu shehuixue sixiang bijiao [Dawn in the Search for Belief - Comparing the Art and Social Science Thought of Confucius and Dewey]," Kongzi Yanjiu 4 (1998): 47-56.

117. Xia Jun, "Duwei shiyongzhuyi lilun yu Zhongguo xiangcun jianshe yundong [Dewey's Pragmatist Theory and China’s Rural Construction Movement]," Minguo Dang'an 3 (1998): 63-68.

118. Ver Zhu Yingxue, "Mao Zedong yu Duwei deyu sixiang bijiao [A Comparison of the Ideas on Moral Education of Mao Zedong and Dewey]," Sanxia Xueyuan Xuebao 1 (2002): 94-97.

119. Zhang Wenhai se refiere explícitamente a esta agenda programática: Zhang Wenhai, "Duwei jiaoyu sixiang dui woguo dangdai hexie jiaoyu de qishi [The Implications of Dewey's Educational Ideas for China's Modern Harmony Education]," Yancheng Gongxueyuan Xuebao (Shehui Kexueban) 4 (2008): 57-59.

120. Yu, "Duwei deyu sixiang dui wo guo xiandai deyu de qishi," 279.

121. Yao Jun, "Qiantan Duwei de deyu fangfa [A Preliminary Investigation of Dewey's Moral Education Methods]," Xiandai Jiaoyu Kexue 1 (2009): 71.

122. Dan Zhonghui, "Lun Duwei de xin de zhiye jiaoyuguan [Discussing Dewey's New View on Vocational Education]," Zhijiao Luntan 7 (2002): 11.

123. Wei Xuefang, "Cong Duwei zhiye jiaoyu sixiang fansi wo guo dangqian zhiye jiaoyu [Reflecting on Our Country's Present Vocational Education through Dewey's Vocational Educational Thought]," Guangxi Jiaoyu Xueyuan Xuebao 6 (2007): 156. 
124. Véase el artículo crítico de Huang Lizhi, "Duwei: Ziyouzhuyi zhi ming yu shi [Dewey: Name and Reality of Liberalism]," Shuwu 3 (2002): 44-46.

125. Ver Tong Dezhi, "Xin jiu ziyouzhuyi — Duwei yu ziyouzhuyi de lilun zhuanxing [Old and New Liberalism - Dewey and the Transformation of Liberalist Theory]," Zhejiang Xuekan 5 (2005): 116-20. 
